\title{
LQAC-5: The fifth release of the Large Quasar Astrometric Catalogue
}

\section{A compilation of 592809 objects with 398697 Gaia counterparts *}

\author{
J. Souchay ${ }^{1}$, C. Gattano ${ }^{2}$, A. H. Andrei ${ }^{1,3,4}$, D. Souami ${ }^{1,5}$, B. Coelho ${ }^{6}$, C. Barache ${ }^{1}$, F. Taris $^{1}$, \\ N. Secrest ${ }^{7}$, and A. Berthereau ${ }^{1}$
}

\author{
1 SYRTE, Observatoire de Paris, PSL Research University, CNRS, Sorbonne Universités, UPMC Univ. Paris 06, LNE, Paris, France \\ e-mail: Jean.souchay@obspm.fr \\ ${ }^{2}$ Laboratoire d'astrophysique de Bordeaux, Univ. Bordeaux, CNRS, B18N, Allée Geoffroy Saint-Hilaire, 33615 Pessac, France \\ 3 Observatorio Nacional/MCT, Rio de Janeiro, Brazil \\ 4 Observatorio do Valongo, UFRJ, Rio de Janeiro, Brazil \\ 5 NAXYS, University of Namur, Rempart de la Vierge, Namur 5000, Belgium \\ ${ }^{6}$ Instituto de Telecomunicações, Campus Universitário de Santiago, Aveiro, Portugal \\ 7 US Naval Observatory, 3450 Massachusetts Avenue NW, Washington, DC 20392, USA
}

Received 21 December 2018 / Accepted 7 February 2019

\begin{abstract}
Context. In addition to their great astrophysical interest, quasars represent quasi-ideal reference objects in the celestial sphere with, a priori, a lack of significant proper motion. Since the fourth release of the Large Quasar Astrometric Catalogue (LQAC-4), a large number of quasars have been discovered, in particular those coming from the DR14Q release of the SDSS. With the advent of the Gaia Data Release 2 (DR2), it is now also possible to fold in extremely accurate quasar positions.

Aims. Following the same procedure as in the previous releases of the LQAC, our aim is to compile the large majority of the recorded quasars, with their best estimated coordinates and substantial information about their physical properties such as the redshift, multibands apparent, and absolute magnitudes. Emphasis is given to the results of the cross-matches with the Gaia DR2 catalogue, which considerably increases the positional accuracy.

Methods. New quasars from the SDSS DR14Q release were cross-matched with the precedent LQAC-4 compilation with a 1" search radius, which leads to 149084 objects not present in the previous LQAC-4 release. Another cross-match was done with the Gaia DR2 catalogue, which enables us to considerably improve the positioning of these objects. For the first time, parallaxes and proper motions from the DR2, when available, are added to our compilation. Furthermore, a cross-identification of the LQAC-5 with the AllWISE survey gives additional mid-infrared information for an important percentage of objects.

Results. Our final catalogue, namely the LQAC-5, contains 592809 quasars. This represents roughly a 34\% increase with respect to the number of objects recorded in the LQAC-4. Among them, 398697 objects were found in common with the Gaia DR2, within a $1^{\prime \prime}$ search radius. That corresponds to $67.26 \%$ of the whole population of the compilation.

Conclusion. The LQAC-5 delivers a nearly complete catalogue of spectroscopically confirmed quasars (including a small proportion of 14126 compact AGN's) to the astronomical community, with the aim of giving their best equatorial coordinates with respect to the ICRF2 and with exhaustive additional information. For more than $50 \%$ of the sample, these coordinates are extracted from the very recent Gaia DR2.
\end{abstract}

Key words. astrometry - quasars: general - reference systems - catalogs

\section{Introduction}

The definition of a quasar is not clearly established. It varies according to the authors, as stated in some detail for instance in Souchay et al. (2015), in particular by comparing the definition given by Véron-Cetty \& Véron (2010) in their compiled catalogue and that given for the SDSS (Pâris et al. 2014). A detailed summary of the typical physical properties of the quasars is given by Proft \& Wambsganss (2015) in the frame of their exploration of these specific objects with the Gaia mission. They are described as Type I active galactic nuclei (AGNs) with line widths of several tens of Angstroms in the quasar rest frame, fol-

\footnotetext{
* The catalogue is available at the CDS via anonymous ftp to cdsarc.u-strasbg.fr (130.79.128.5) or via http://cdsarc. u-strasbg.fr/viz-bin/qcat?J/A+A/624/A145
}

lowing a standard model proposed by Urry \& Padovani (1995). This model consists of a super-massive black hole (SMBH) in the centre of an accretion disc surrounded by an opaque torus, with in a close vicinity a broad line region (BLR) and farther a narrow line region (NLR), named from observed spectral features, and accompanied by jets detected at radio wavelengths. This unified model is constantly under minor revisions as observations continue and are improved. Recently, Padovani et al. (2017) gave a review on AGN physical properties interpreted from multi-wavelength observations and in the context of the unified model.

In addition to their great astrophysical interest, the compactness of the quasars as well as their lack of proper motion make them ideal for astrometry. Quasars represent the basis of modern astrometry as they are supposed to give quasi-fixed 
directions in the universe from our Earth-centred point of view. For this property, they are natural candidates with which to materialise the International Celestial Reference System (ICRS, Arias et al. 1995) as a quasi-inertial reference frame, namely the ICRF. The ICRF is used for various scientific studies in the fields of fundamental physics (Le Poncin-Lafitte et al. 2016a,b), geodesy and geophysics (Gattano et al. 2017; Rosat et al. 2017) or solar system exploration. the second version (Fey et al. 2015) contains 3414 objects of which the large majority are quasars. A new version was adopted by the IAU General Assembly at Vienna in August 2018 and in preparation for publication. In parallel with these new developments, and with the data obtained from the Gaia mission, efforts were made to build a new rotation-free celestial reference frame in the visible wavelengths, called the Gaia Celestial Reference Frame (Gaia-CRF) meeting the specifications of the International Celestial Reference System (Arias et al. 1995). For this purpose we can refer to Gaia Collaboration (2016a) and to Mignard et al. (2018) for the latest realisation, Gaia-CRF2.

From these considerations, it appears that the compilation of all the spectroscopically confirmed quasars, at a given date, in various large surveys and quasar catalogues is of fundamental utility for the astronomical community. Besides getting the location of all the recorded quasars within the celestial sphere, such a compilation gathers various information on each of them, thanks to the input of each catalogues taken in consideration. For examples, redshift, multi-wavelengths, optical, and infra-red magnitudes, as well as radio-fluxes. Moreover, it enables complementary studies, such as the investigation of offsets between the positions given by the original catalogue and those given by an improved astrometric determination, as well as sky coverage statistics (Gattano et al. 2014).

Over the last decade, efforts have been made to compile all the recorded quasars with the fundamental purpose of giving the, a priori, best value for their equatorial coordinates. The resulting Large Quasar Astrometric Catalogues (LQAC) were updated on a regular basis, in particular by including at each release a large amount of newly discovered quasars that came from successive SDSS releases. A decade ago, the fist version of the LQAC (Souchay et al. 2009) contained 113666 objects, whereas the last version - the LQAC-4 (Gattano et al. 2018) - includes 443725 objects.

In LQAC-4, considerable improvements have been achieved thanks to the use of the Gaia DR1 release (Lindegren et al. 2016) which provides for the first time the equatorial coordinates of a very large number of quasars at the level of the milliarcsecond (mas) accuracy or better. Before, this was only achieved for a few thousands of sources and only in radio thanks to the Very Long Baseline Interferometry (VLBI), whereas among the 443725 objects of the LQAC-4, 249017 were found in correspondence with one object of the DR1, i.e., $56.13 \%$ of the total number.

Mignard et al. (2018) underline that Gaia is meant, by the end of the mission, to be autonomous in terms of the recognition of quasars from their photometric properties (colours, variability). Nevertheless, this functionality had not yet been implemented for the first two releases and therefore the sources currently identified as quasars are drawn from other quasar catalogues, cross-matched with Gaia sources.

With respect to the LQAC-4 (Gattano et al. 2018), the updated version LQAC-5 presented in this paper constitutes a real progress in a quantitative and qualitative point of view. First, it contains a significant number of new quasars, due to the introduction of the last release of the SDSS quasar survey DR14Q
(Pâris et al. 2018). Second, it is cross-matched with the Gaia DR2 (Lindegren et al. 2018) improving the astrometric quality of the compilation as mentioned above and, for the first time, a determination of the parallax and proper motions. Third, the LQAC-5 is cross-matched with the WISE survey from which mid-infrared multi-band magnitudes are available for a large percentage of objects.

In this paper, we describe in details the characteristics of the LQAC-5 catalogue and its improvements with respect to the LQAC-4. In Sect. 2, we examine the characteristics of the new quasars which are introduced in the LQAC-5. In Sect. 3, we analyse the improvement from the Gaia-DR2 in a qualitative and quantitative point of view. In Sect. 4, we present the new contribution of the AllWISE survey in the LQAC compilation. In Sect. 5, we focus on the procedure that derives accurate equatorial coordinates of the quasars not observed by Gaia based on the LQRF (Large Quasar Reference Frame) algorithms. Section 6 is devoted to the calculation of the absolute magnitudes of the quasars, with the intermediary of a recent method which had already been used in the LQAC-4 release. The advantageous specificities of the LQAC-5 compilation of quasars presented in this paper must be emphasised with respect to the recent catalogues of AGN as the WISE AGN catalogue (Assef et al. 2018). and the Gaia-WISE extragalactic astrometric catalogue (Paine et al. 2018), both being based on two-colours diagrammes. Section 7 concerns the calculation of morphological indexes. Finally the quantitative and qualitative advantages of the LQAC-5 are discussed in the conclusion in Sect. 8.

\section{New contribution of the DR14Q SDSS release}

The quasars of the LQAC-5 not included in the LQAC-4 are extracted exclusively from the Sloan Digital Sky Survey (SDSS) data release 14 Quasar catalogue (DR14Q, Pâris et al. 2018), which is extracted from the extended Baryon Oscillation Spectroscopic Survey (eBOSS) of the SDSS stage IV (SDSS-IV). The DR14Q includes all SDSS-IV/eBOSS objects that were spectroscopically targeted as quasar candidates and that are confirmed as quasars via a robust automated identification procedure combined with a partial visual inspection of the spectra of about $10 \%$ of their sample identified as ambiguous candidates. All these objects have been selected using a well established rule: their absolute magnitude $M_{i}[z=2]$ must be smaller than -20.5 in the frame of the conventional cosmology with $H_{0}=70 \mathrm{~km} \mathrm{~s}^{-1} \mathrm{Mpc}^{-1}, \Omega_{\mathrm{M}}=0.3$, and $\Omega_{\Lambda}=0.7$. Moreover, they must either display at least one emission line with a full width at half maximum larger than $500 \mathrm{~km} \mathrm{~s}^{-1}$ or have interesting and complex absorption features. The redshift measurements are based on a principal component analysis of the spectra. According to Pâris et al. (2018), the DR14Q is estimated to have about $0.5 \%$ contamination. Some quasars have been observed multiple times throughout the 16 years of the survey.

We note that the DR14Q contains about $80 \%$ more quasars at $z<2$ than the previous release (Pâris et al. 2017). Whereas the average surface density of $0.9<z<2.2$ quasars prior to the beginning of SDSS-IV was 13.27 per $\mathrm{deg}^{2}$, it reaches 80.24 per deg $^{2}$ in regions for which SDSS-IV spectroscopy is available. The DR14Q includes 526356 quasars, of which 144046 are new discoveries. This represents an increase of about $40 \%$ in the number of SDSS quasars since the beginning of SDSS-IV campaign.

Spectroscopic observations of quasars were performed over $9376 \mathrm{deg}^{2}$ for SDSS-I/II/III. New SDSS-IV spectroscopic data are available over an additional $2044 \mathrm{deg}^{2}$. The overall quasar 
surface density in regions with SDSS-IV spectroscopy is 125.03 per $\mathrm{deg}^{2}$, which corresponds to an increase by a factor of 2.4 times compared to the previous SDSS quasar catalogue release.

Finally, we note that the various successive campaigns of the SDSS were focused on different redshifts intervals: SDSS-I/II (York et al. 2000) has observed quasars in the range $0<z<$ 5.4 with a nearly flat distribution up to $z \sim 2.5$, followed by a steep decrease. SDSS-III (Eisenstein et al. 2011) has focused on $z>2.15$ in order to access the Lyman- $\alpha$ forest. SDSS-IV/eBOSS mostly aimed to fill the gap between $z=0.8$ and $z=2$. In the distribution of redshifts, two peaks are easily identified at $z=0.8$ and $z=1.6$. This is explained by known degeneracies in the associated quasar target selection Ross et al. (2012).

\section{Benefits from the recent Gaia DR2 data}

The first data release from Gaia observations (DR1) was produced in September 2016 (Lindegren et al. 2016) after 14 months of nominal operations. Amongst the 443725 objects of the LQAC-4 (Gattano et al. 2018), 249071 were found in common with the Gaia DR1 within a 1" search radius, providing a considerable improvement on their equatorial coordinate accuracy with respect to data from other original catalogues, such as the SDSS.

On April 25th, 2018, Gaia DR2 was made available on the dedicated ESA open-access online archive ${ }^{1}$. It results from the integration of the 22 first months of the satellite nominal operations. This second official astrometric solution (Lindegren et al. (2018) benefits from several improvements. First of all, the satellite attitude model was refined in such a way that microclanks and micro-meteoroid impacts were corrected without any consequent degradation to the astrometric high efficiency of the instrument. Secondly, the calibration model was continuously improved to take into account the unavoidable changes of the optical and mechanical structure of the instruments in the extreme environment of the outer space and necessary maintenance operations. Particular care was made to compensate the perturbation observed on the basic angle (mainly related to the six-hour heliotropic spin of the satellite) propagating into the data. This calibration model also took into account the scanning law of the satellite in order to avoid degradation due to the calibration of the celestial reference frame. In this context, the expected Gaia photometric range of quasars, roughly $G$-mag $\sim 15$ and fainter, is the range least affected by mis-modelling or non-modelled effects.

The Gaia-DR2 release contains astrometry, broad-band photometry, radial velocities, and the characteristics of the corresponding light-curves for a total of 1692919135 sources. This represents a $48.16 \%$ increase with respect to the total number of 1142679769 in the Gaia-DR1. The methodology behind these computations can be summarised as follows: (i) Preliminary solutions were made to retrieve initial data for all available sources and satellite-related model parameters. (ii) A set of primary sources was selected for their good quality as revealed by the preliminary solutions, including quasars for the orientation of the solution on the ICRS. (iii) A first secondary solution focusing on the limited but still large set of primary sources established data for the attitude and calibration models. (iv) The solution was oriented onto the ICRS. (v) The second secondary solution - the final one - considered the satellite attitude and the

\footnotetext{
1 Link to the web page of the Gaia archive $\rightarrow$ http://gea. esac. esa.int/archive/
}

calibration as known and adjusts data for all the 1692919135 available sources. We caution that this is only a rough summary of the Gaia collaboration works. In practice, they led several studies and developed various solutions enabling them to identify several sources of errors in the modelling strategy. We refer the reader to Lindegren et al. (2018) and references therein for details concerning the procedure.

For the DR2, the Gaia collaboration was not able yet to identify quasars internally (Delchambre 2018). This ability will be unlocked in the future. In the meantime, quasars within the Gaia catalogue were identified by means of cross-identification with other external catalogues. The Large Quasar Astrometric Catalogue is perfectly suited to this purpose.

\subsection{Astrometric improvement in LQAC-5: Gaia DR2 with respect to Gaia DR1}

The cross-identification between catalogues is a delicate task. The same quasars in two different catalogues show slightly different equatorial coordinates. Thus a search radius is necessary to identify identical objects in the two catalogues. The subtlety lies in the choice of search radius. If the radius is too small $(\sim 0.1$ arcsec), there is a high probability of missing the object in the other catalogue. On the other hand, if it is too large $(\sim 10 \operatorname{arcsec})$, the probability of finding a wrong counterpart becomes large. We studied the inter-dependency of the number of cross-identifications between LQAC-4 and Gaia DR1 with respect to the value of the search radius $\rho$ (Gattano et al. 2018). We show that the number of cross-matches increases very slowly beyond the value of $\rho=1^{\prime \prime}$. This increase is less than $3 \%$ between $\rho=1^{\prime \prime}$ and $\rho=3^{\prime \prime}$. We adopted the $\rho=1^{\prime \prime}$ threshold for the cross-identifications involved in the present LQAC-5.

The number of cross-matches with Gaia DR2 data increases significantly with respect to LQAC-4 which was confronted to the DR1. This is due both to the inclusion of the 144046 new quasars of the SDSS DR14Q detailed in the previous section, and to the fact that the DR2 release itself contains substantially more objects than the DR1, with a $48.16 \%$ increase. Moreover, LQAC5 also benefits from a drastic improvement of the uncertainty in the determination of the equatorial coordinates, when obtained by Gaia. This means that for a large percentage of quasars in the LQAC-5 we now know their position to the milliarcsecond level of accuracy, whereas only the 3414 sources in the ICRF2 reached this accuracy. Finally, Gaia-DR2 provides parallaxes and proper motion on right ascension and declination, with a generally very small formal error.

To emphasise the improvement in the determination of the source equatorial coordinates from the DR2 with respect to the DR1, in Fig. 1 we plot the associated uncertainties for the 248365 quasars common to the two releases. For a significant proportion of quasars, this uncertainty has decreased by one order of magnitude, from typically the mas level to a fraction of mas level. This drastic amelioration is easily confirmed by corresponding histograms (see Fig. 2). In right ascension, the histograms peak at an uncertainty of 0.3 mas for the DR2 and an uncertainty of 0.5 mas for the DR1. Moreover, we note a very flat feature for the DR1 distribution, and no feature for the DR2 one. Analogous remarks can be made in declination. Notice that the uncertainty in declination is globally smaller than that in right ascension, the number of objects ranging in the interval $[0.0,0.3]$ mas being significantly larger. From Fig. 1 we also see that for a small number of quasars, positions in DR2 are worse than in DR1. This fact can be explained by magnitude variation: Quasars should become dimmer in the second release 

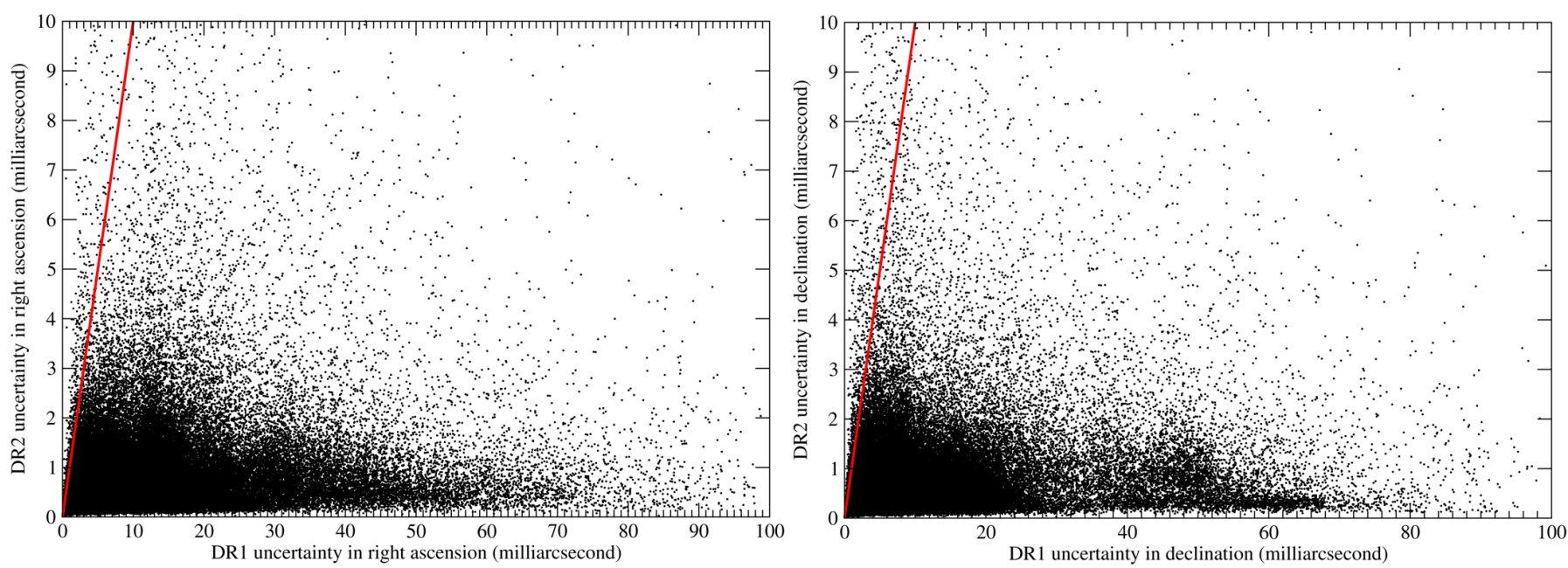

Fig. 1. Comparison of equatorial coordinate accuracies from the two Gaia releases. Left panel: diagram of the DR2 uncertainty with respect to the DR1 uncertainty in right ascension for the 248365 quasars of the LQAC-5 belonging to both releases. Right panel: same diagram in declination. The red straight line indicates equality between uncertainties from the two releases.
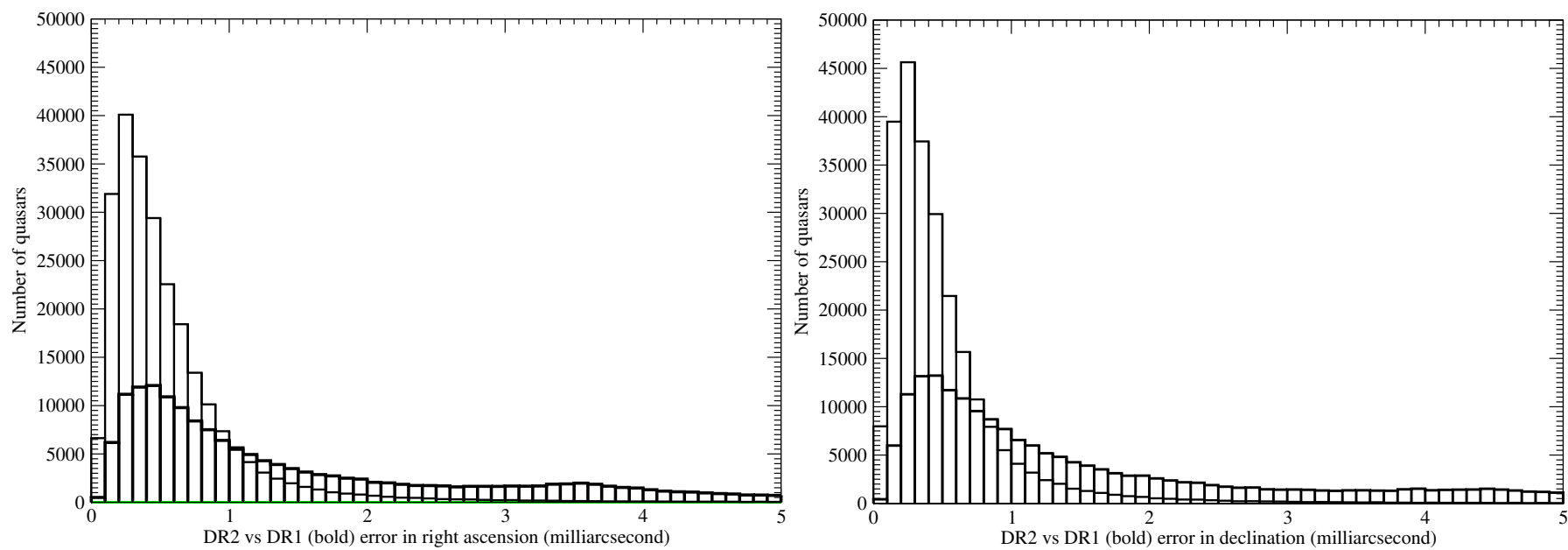

Fig. 2. Left panel: histograms of the uncertainties from Gaia DR2 and Gaia DR1 (bold) in right ascension for the 248365 quasars of the LQAC-5 belonging to both releases. Right panel: same histograms in declination.

or show some photocentre jitters. Another reason arises from the complexity of the Gaia procedure which tends to divide the observations in small groups, which can give statistically larger uncertainties in the second release than in the first.

The differences in coordinates between the DR1 and DR2 are given in Fig. 3. For a large majority of objects we find that the absolute value of these differences is smaller than 1 mas, but that a significant percentage of objects have larger values. Here, still, the differences are significantly smaller in declination than in right ascension. Finally, Fig. 4 shows the angular distances between the DR2 and the DR1 positions. We observe a rapid decrease after a peak at 0.3 mas. Nevertheless, the number of sources for which the angular distance is larger than 1 mas is significant.

\subsection{Photometric comparisons between Gaia DR1 and DR2}

In the previous LQAC-4 release (Gattano et al. 2018), the Gaia $G$ band measurements were available and included for the 249017 quasars found in common with the DR1. Nevertheless, as mentioned by Gaia Collaboration (2016b), the $G$-band fluxes and magnitudes provided with the DR1 have standard uncer- tainties as good as a few per cent in magnitude at the survey limit and down to the milli-magnitude level at the bright end. Indeed there are limitations inherent to the DR1. In particular, there is a small fraction of sources well beyond the Gaia limit of $G=20.7$ for which the mean value of $G$ is clearly wrong. More precisely the DR1 potential systematic errors for the determination of $G$ are described in Evans et al. (2017) and Arenou et al. (2017).

As mentioned by Gaia Collaboration (2018) the photometric data processing for Gaia DR2 features many improvements with respect to Gaia DR1 and represents a new photometric reduction. In particular, the data was considerably extended and some technical problems related to the contamination by water ice were removed. As a result, the photometric system for Gaia DR2 is different from that for Gaia DR1. The comparison between the $G$ magnitudes by taking into account pairs of objects belonging to the two releases and presenting a $G_{\mathrm{BP}}-G_{\mathrm{RP}}$ colour determination was quantified by Gaia Collaboration (2018) the following polynomial relation:

$$
\begin{aligned}
\Delta G= & G_{\mathrm{DR} 2}-G_{\mathrm{DR} 1}=0.013612+0.079627\left(G_{\mathrm{BP}}-G_{\mathrm{RP}}\right) \\
& +0.004044\left(G_{\mathrm{BP}}-G_{\mathrm{RP}}\right)^{2}-0.0018602\left(G_{\mathrm{BP}}-G_{\mathrm{RP}}\right)^{3} .
\end{aligned}
$$



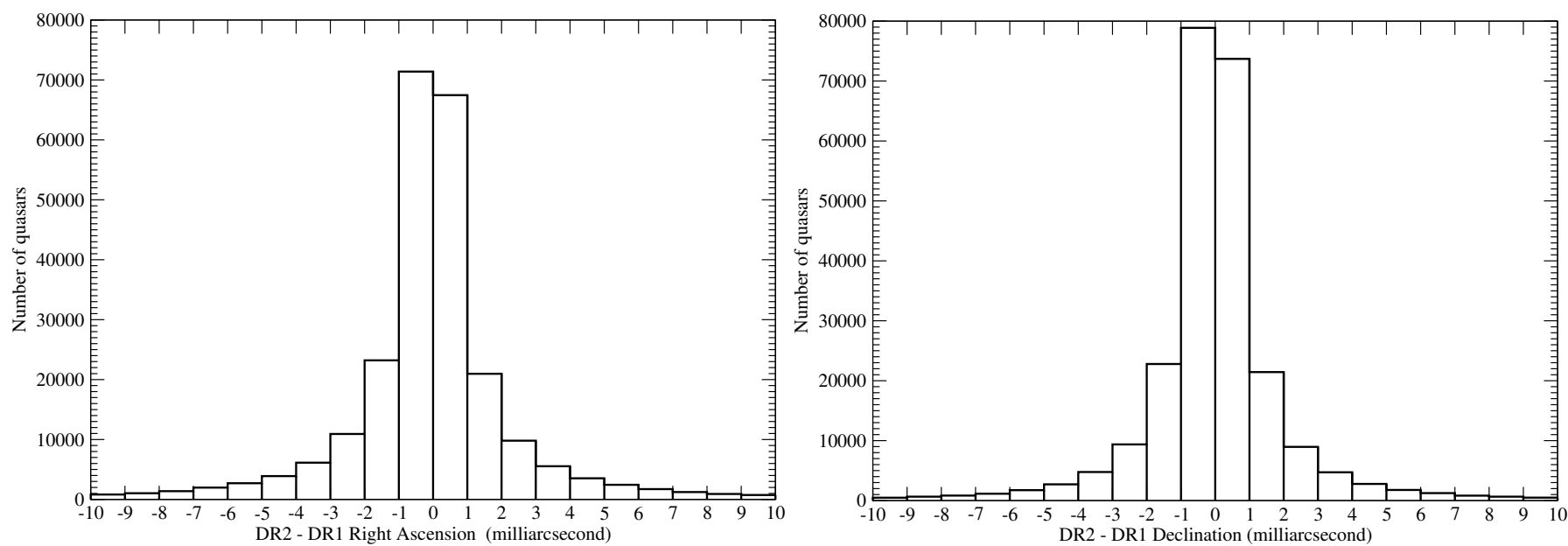

Fig. 3. Source position offsets from the two Gaia releases. Left panel: histogram of the source right ascension offset between Gaia DR2 and Gaia DR1 for the 248365 quasars of the LQAC-5 belonging to both releases. Right panel: same histogram in declination.

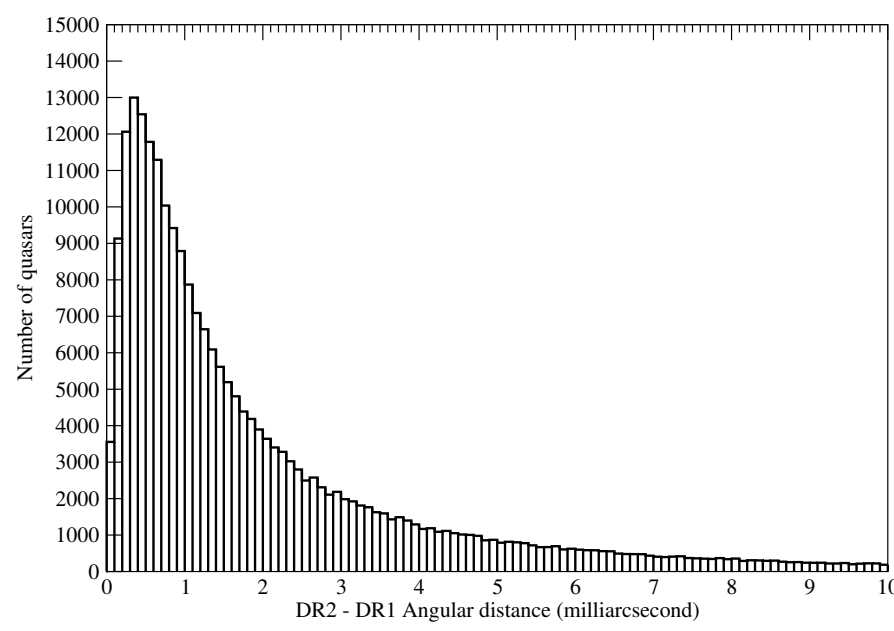

Fig. 4. Source position offsets from the two Gaia releases. The histogram shows the angular distances between Gaia DR2 and Gaia DR1 for the 248365 quasars of the LQAC-5 belonging to the two releases.

In our study we have carried out such a comparison for the quasars in common to the two releases. Results are shown in Fig. 5. We found $\Delta G=0.0911$ with a standard deviation $\sigma_{\Delta G}=$ 0.139 , considerably larger than the bias given in Eq. (1), so that it appears clearly in the histogram. Notice that as the majority of quasars are variable in a rather short time scale, sometimes at the level of several $0.1 \mathrm{mag}$, and given the rather large time interval between the two releases, of the order of one and a half year, significant standard deviation $\sigma_{\Delta G}$ could originate in a non negligible part from the variability of the objects.

We also investigated the fundamental question of GaiaDR2 completness. All quasars brighter than $G=20$ should be detected by Gaia. To determine the completeness of Gaia-DR2, we concentrated our investigation on the SDSS-known objects, for the sake of homogeneity. Although we do not have the strict equivalent of the $G$ band in the SDSS data, the SDSS magnitude $r$ is very close to $G$. As explained by Jordi et al. (2010) the Gaia $G$ band has a central wavelength at $\lambda_{0}=673 \mathrm{~nm}$ and a FWMH $\Delta \lambda=440 \mathrm{~nm}$. In parallel, the SDSS $r$ band corresponds to $\lambda_{0}=620 \mathrm{~nm}$ and a FWMH $\Delta \lambda=113 \mathrm{~nm}$. Thus, we observe that the central wavelengths are very close, and despite the relatively large difference in bandwidth, we note a good agreement

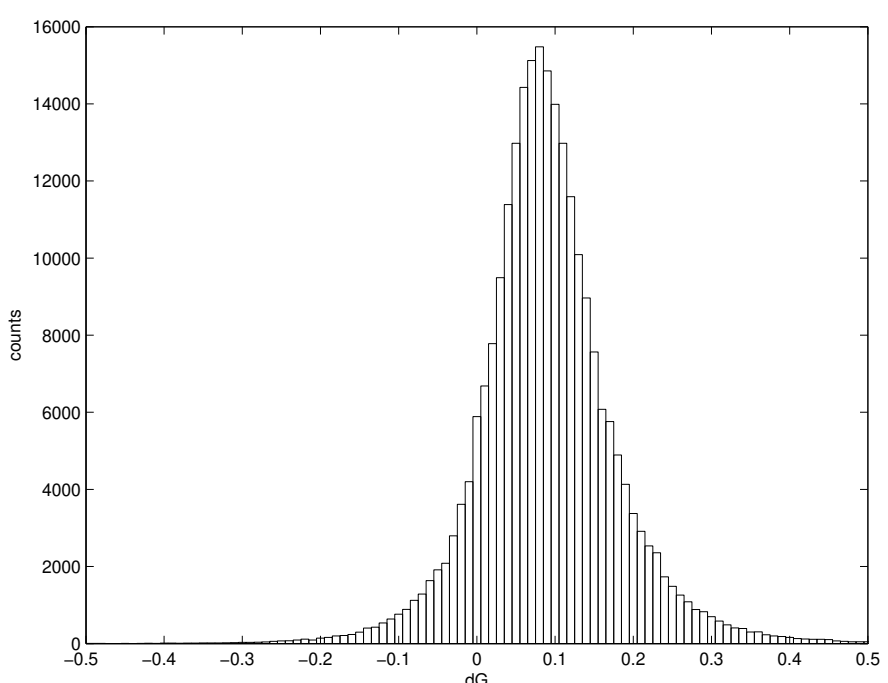

Fig. 5. Photometric comparison from the two Gaia releases. Histogram of the differences $\Delta G=G_{\mathrm{DR} 2}-G_{\mathrm{DR} 1}$ for the quasars in common in the two releases.

between $G$ (Gaia) and $r$ (SDSS). As shown by Gattano et al. (2018) from the DR1, values generally stand within the interval $\pm 1 \mathrm{mag}$.

By consulting the Table 1 illustrated by the histogram in Fig. 6, on the basis of the SDSS known population in LQAC-4 and LQAC-5, Gaia-DR2 is found nearly complete while this is far from being acquired with the DR1. For the brightest quasars, with $15<r<18$ the number of DR2 identifications attains respectively 8378 objects among the 8390 ones in the SDSS of the LQAC-5, which represents $99.85 \%$ of the sample, with only 12 objects lacking. These missing objects require a specific individual inspection to understand why they are missing. In comparison, in the same interval of magnitude, 7816 quasars are reckoned in the DR1 among the 8088 objects of the SDSS which belonged to the LQAC-4. This represents only $96.69 \%$ of the sample with 272 lacking objects. The difference of completeness between DR1 and DR2 is even more important when we look at the faint objects in $r$. For instance in the interval $20<r<20.5$ only $75.76 \%$ of the SDSS quasars are present in the DR1, whereas the percentage reaches $96.6 \%$ for the DR2. The percentage of DR2 detections remains quite large for the 
Table 1. Number of quasars of the DSS present in the DR1 and in the DR2 (with the corresponding percentage, with respect to their $r$ SDSS magnitude.

\begin{tabular}{lrrrrrr}
\hline \hline$r$ interval & SDSS in LQAC-4 & In DR1 & \% in DR1 & SDSS in LQAC-5 & In DR2 & \% in DR2 \\
\hline $15.0-15.5$ & 12 & 12 & 100.00 & 15 & 13 & 86.67 \\
$15.5-16.0$ & 70 & 67 & 95.71 & 74 & 72 & 97.30 \\
$16.0-16.5$ & 185 & 177 & 95.67 & 196 & 195 & 99.49 \\
$16.5-17.0$ & 629 & 607 & 96.50 & 649 & 645 & 99.38 \\
$17.0-17.5$ & 1858 & 1805 & 97.20 & 1915 & 1908 & 99.63 \\
$17.5-18.0$ & 5334 & 5148 & 96.51 & 5594 & 5577 & 99.70 \\
$18.0-18.5$ & 14077 & 13542 & 96.20 & 15060 & 15036 & 99.84 \\
$18.5-19.0$ & 31168 & 29648 & 95.12 & 33616 & 33525 & 99.73 \\
$19.0-19.5$ & 45479 & 42779 & 94.06 & 51957 & 51766 & 99.63 \\
$19.5-20.0$ & 48495 & 43951 & 90.63 & 62396 & 62013 & 99.39 \\
$20.0-20.5$ & 64206 & 48645 & 75.76 & 87558 & 84479 & 96.48 \\
$20.5-21.0$ & 63655 & 25118 & 39.46 & 99133 & 74590 & 75.24 \\
$21.0-21.5$ & 58845 & 5908 & 10.04 & 96839 & 29750 & 30.72 \\
$21.5-22.0$ & 40691 & 699 & 1.72 & 69073 & 4659 & 6.74 \\
$22.0-22.5$ & 8455 & 59 & 0.70 & 15081 & 359 & 2.38 \\
$22.5-23.0$ & 537 & 3 & 0.56 & 1269 & 28 & 2.21 \\
$23.0-23.5$ & 70 & 1 & 1.43 & 171 & 8 & 4.68 \\
$23.5-24.0$ & 21 & 1 & 4.76 & 33 & 2 & 6.06 \\
\hline
\end{tabular}

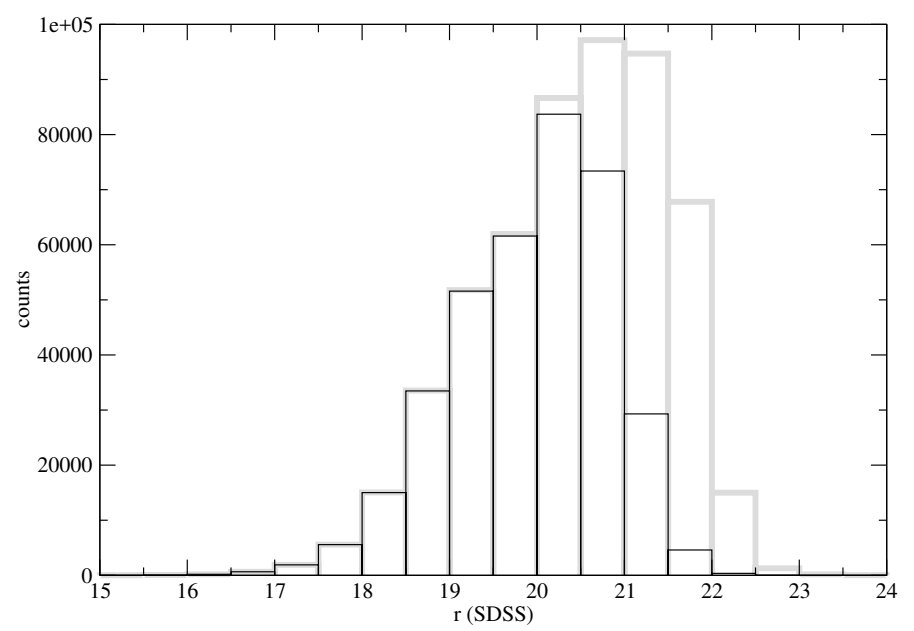

Fig. 6. Number of SDSS quasars present in the Gaia DR2 release (in black) with respect to the total distribution (in grey), per bin of $r$ magnitude.

intervals $20.5<r<21(75.54 \%)$ whereas it is nearly twice as small with the DR1 $(39.46 \%)$.

From these statistics we can conclude that the completeness of DR2 detection for $r<20$ is very satisfactory, with a detection rate of more than $99 \%$ and that the large differences of completeness between the DR1 and the DR2 could be interpreted as either a deficiency inherent to the DR1 detection capability, the lack of completeness of the Gaia scanning itself, or an improved detection threshold in the DR2 with respect to DR1 due to an increase of information or a decrease of the noise level.

\section{Cross-identification with the AlIWISE survey}

The Wide-field Infrared Survey (WISE, Wright et al. 2010) is an all-sky mid-IR survey at 3.4, 4.6, 12, and $22 \mu \mathrm{m}$ (W1, W2, W3, and W4 respectively) conducted in 2010 and made available to the public in April 2011. The AllWISE output catalogue contains positions, apparent motions, magnitudes, and point-spread function (PSF)-profile fit information for about 748 million objects. Moreover, AllWISE also contains aperture magnitudes and elliptical magnitudes for objects that are also in the 2MASS Extended Source Catalogue (XSC). We note that the astrometric treatment of the objects was done starting both from the bright objects from the 2MASS point source catalogue and the fourth USNO CCD Astrograph Catalogue (UCAC4, Zacharias et al. 2013). Specifically, proper motions from UCAC4 were used to propagate the $2 \mathrm{MASS}$ counterpart positions to the epoch of WISE.

As we are in the mid-infrared zone, the angular resolutions corresponding to the four successive bandwidths above are 6.' 1 , 6.' 4,6 .' 5 and 12 '. 0 respectively. For the cross-identifications we used a $5^{\prime \prime}$ match radius, which was chosen as being a balance between reliability and completness. However, we noticed an AllWISE colour dependence (W1-W2, W2-W3) of the match distances. Generally, sources with colours more like starburst galaxies (redder in W2-W3 and bluer in W1-W2) have a larger match distance. This is likely, as they tend to be somewhat extended. Sources with more pure QSO-like colours have a much tighter distribution of match distances; these are generally unresolved. In total we find 488736 cross-identifications in the $W_{1}$ and $W_{2}$ bands, 488599 in the $W_{3}$ band and 488597 in the $W_{4}$ band. This corresponds respectively to $82.44 \%$ and $82.42 \%$ of the whole LQAC5 population.

\section{Determination of LQRF coordinates}

There are 360664 quasars belonging to the SDSS that are unmistakably found in the Gaia DR1 and DR2 releases. Of those, only 124 appear in the DR1 alone, which has a negligible deviation of its reference frame with respect to the one of the DR2. Therefore, in the following discussion, no distinction between the two Gaia data releases is required.

As illustrated previously in Fig. 6, we count 172296 quasars belonging to the SDSS but not present in the Gaia two early releases, within the adopted 1 " search radius. Most of them are 
faint objects, escaping to the detection by Gaia, and a few of them may be too extended to be yet detected as a single object by the Gaia point source algorithm. Nonetheless, quite a few of them are well behaved QSOs, which may be largely variable, or missed in too-crowded fields, or just being serendipitously so far missed by Gaia. Whichever the case, it is important to place all these objects in the Gaia reference frame. This is done following the precepts used for the construction of the LQRF (Andrei et al. 2009), as well as in the former issues of the LQAC. The construction of the LQRF entails four redressing steps, with which we aim to obtain astrometric coordinates in the Gaia reference system, which is consistent to the ICRF/ICRS. Namely,

(1) a local astrometric solution - which aims to recover the innermost quality of the measure by de-projecting the catalogue coordinates onto the local tangential plane.

(2) an Euclidean rotation relatively to the three Euclidean axes combined to an equatorial bias assessment - which aims to coincide the implicit axis of the catalogue with the Gaia reference frame axis (Andrei et al. 2009; Arias et al. 1988).

(3) a set of harmonic solutions over the sphere - which aims to straighten out large-scale ripples on the right ascension, declination, and magnitude respectively by normalised Legendre polynomials, Fourier series, and Hermite polynomials.

(4) local inhomogeneities - which aim to correct any residual deviation of the equatorial coordinates by comparing the mean offsets of several close-by fiduciary sources.

The SDSS coordinates constitute a tightly coherent catalogue reference frame, despite irregularities noticed by Gattano et al. (2018). So far, the local astrometric solution correction is negligible at the level of a few milli-arcseconds, as already shown in Andrei et al. (2009) and Souchay et al. (2009). This initial step of the standard derivation of LQRF-like coordinates is therefore not used, as it would indeed be bound to the introduction of a random noise rather than to the removal of systematic effects. On the other hand, the SDSS sky coverage is limited and not continuous, making dubious the derivation of large scale quasi-periodic terms. The same happens for the magnitudes in view of the limited range of apparent magnitude of the quasars both in the SDSS itself and in Gaia. The LQRF/Gaia (SDSS), that is, the redressing of the SDSS coordinates onto the Gaia reference frame, is accomplished by steps (2) and (4) above.

The Euclidean rotations and the equatorial biases are derived as in the former LQAC versions, following the methodology found in Arias et al. (1988) and Andrei et al. (2009). For the 360664 sources common to the SDSS and the Gaia two early releases, solutions were obtained both independently for the right ascensions and declinations, as well as to three levels of threshold at 2.0, 2.5, and 3.0 the value of the standard deviation. In all cases, the solutions are concurrent. The numerical values of the direction cosines and the biases are small as expected, though not statistically negligible. Adopting the solution that acknowledges the more equations, the equinox bias is $+8.8 \pm 0.1$ mas and the equatorial bias is $+1.0 \pm 0.1$ mas. The rotation around the equinox oriented $X$-axis is $-6.8 \pm 0.1$ mas, and finally the rotation around the remaining triedal $Y$-axis is $+1.5 \pm 0.1$ mas. The overall standard deviation of the solution amounts to 41.9 mas and the values must be taken in the SDSS towards Gaia direction.

The somehow important equinox and declination biases can be seen in Fig. 7: the histograms of the SDSS minus Gaia equatorial coordinate residuals. For the right ascension distribution, a slight negative asymmetry affects the whole and otherwise
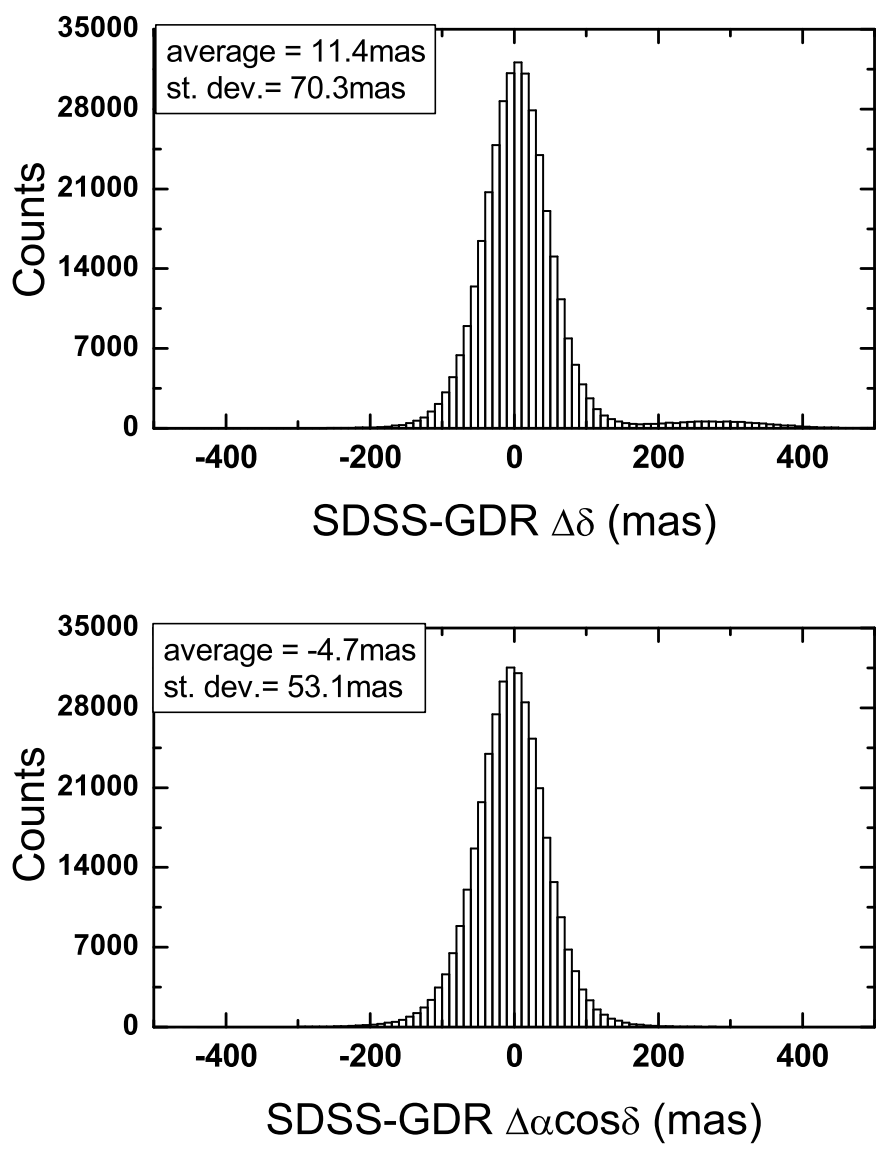

Fig. 7. Comparison of equatorial coordinate residual between SDSS and Gaia. Bottom panel: histogram of the right ascension cosine declination offsets between the Gaia-DR2 and SDSS positions for the quasars in common in the two catalogues. Top panel: same histogram in declination.

clearly bell-shaped distribution. For the declination, the curve is overall a classical bell-shape one, but with a small but statistically real positive tail. For the right ascension, an equator bias is found on the magnitude, redshift, and right ascension offset plots (see Figs. 8 and 9). The tail originates from the equatorial north cap SDSS coordinates (which is related to those SDSS sources which are faint and far away). The number of such sources corresponds to less than $10 \%$ of the total number of SDSS sources, and thus the general biases and rotations are not affected. Notwithstanding, they indicate the usefulness of step (4) of the formation of the LQRF/Gaia (SDSS), that is, the source-by-source correction of local inhomogeneities.

The local inhomogeneities corrections account for the ground catalogues clumping, derived from particular observational conditions, thus without forming a clear pattern across the sky. For each individual SDSS source they are determined by the average right ascension and declination offsets (once rotation and bias are accounted for) of ten or more nearby sources belonging to the SDSS and Gaia catalogues. Nonconforming values are eliminated. In the formation of the LQRF/Gaia (SDSS) this can be studied in detail, and the corresponding corrections applied to the catalogue coordinates, already oriented towards the axis of the Gaia frame, as obtained in the previous step. Accordingly, for each source the average equatorial coordinates offset is determined from the ten closest catalogue sources which do belong to the Gaia early releases catalogues. Offsets that disagree by a threshold of 2.5 standard 


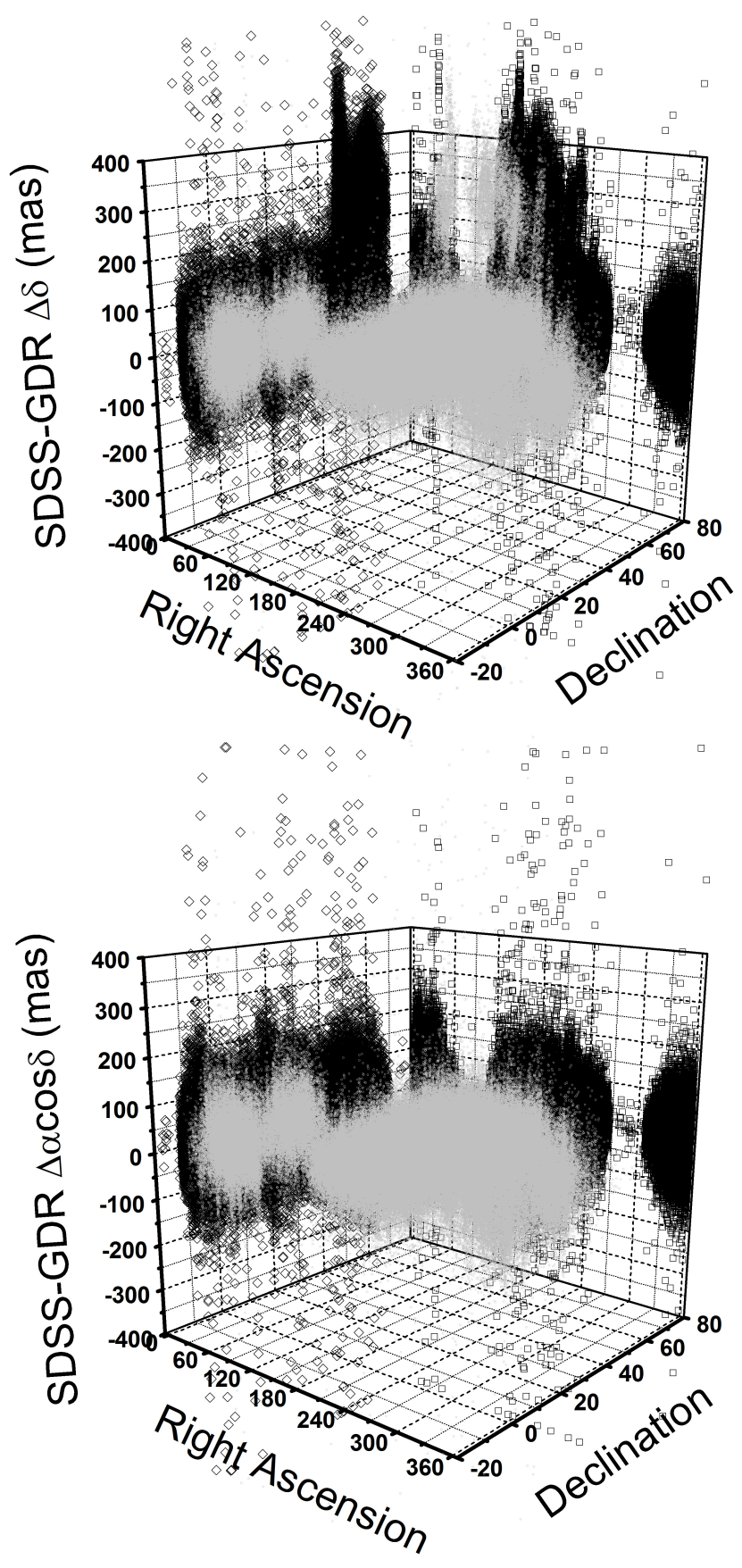

Fig. 8. Comparison of equatorial coordinates between SDSS and Gaia with respect to source position. The grey dots show the (3D) diagram of position offsets between the Gaia-DR2 and SDSS with respect to the Gaia-DR2 source position on the sky for the quasars in common in the two catalogues. Empty black squares show the associated projected diagram (2D) along the Gaia-DR2 right ascension axis. Empty black diamonds show the associated projected diagram (2D) along the GaiaDR2 declination axis, limited to the range $\left[-20^{\circ}, 80^{\circ}\right]$. Bottom panel: right ascension cosine declination part of the offsets. Top panel: declination part of the offsets.

deviations are discarded from the averages. This was the case for $1.5 \%$ of the sources.

The local inhnomogeneities are small, as expected (see Fig. 10). On the right ascension the average is -0.39 mas with a standard deviation of 11.0 mas, and extreme values of -235.2 mas and 229.2 mas. On declination the average is 7.40 mas with a standard deviation of 12.40 mas, and extreme
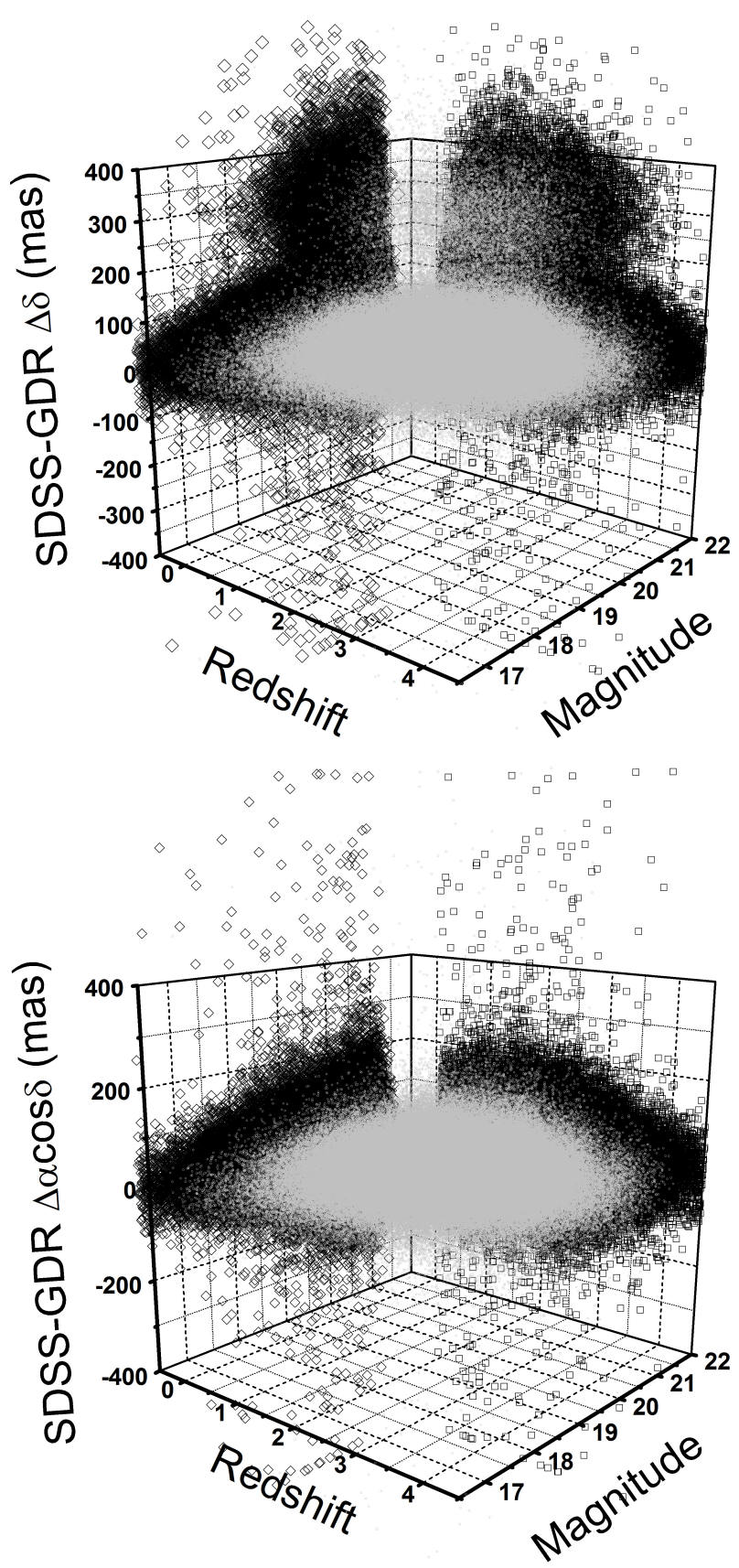

Fig. 9. Comparison of equatorial coordinates between SDSS and Gaia with respect to magnitude and redshift. Grey dots show the (3D) diagram of position offsets between the Gaia-DR2 and SDSS with respect to the redshift and magnitude for the quasars in common in the two catalogues. Empty black squares show the associated projected diagram (2D) along the redshift axis. Empty black diamonds show the associated projected diagram (2D) along the magnitude axis. Bottom panel: right ascension cosine declination part of the offsets. Top panel: declination part of the offsets.

values of -282.6 mas and 512.6 mas. The similarly small right ascension and declination mean values are seen graphically on the histograms, with a clearly Gaussian feature (see Fig. 11).

Forming a vector composed of right ascension and declination corrections, the magnitude frequency histogram and the angular direction histograms also show these characteristics. The corrections are small and non-coherent on a large scale. This is shown on the right ascension plot with respect to the declination one (see Fig. 12). Notwithstanding, over small scales the 

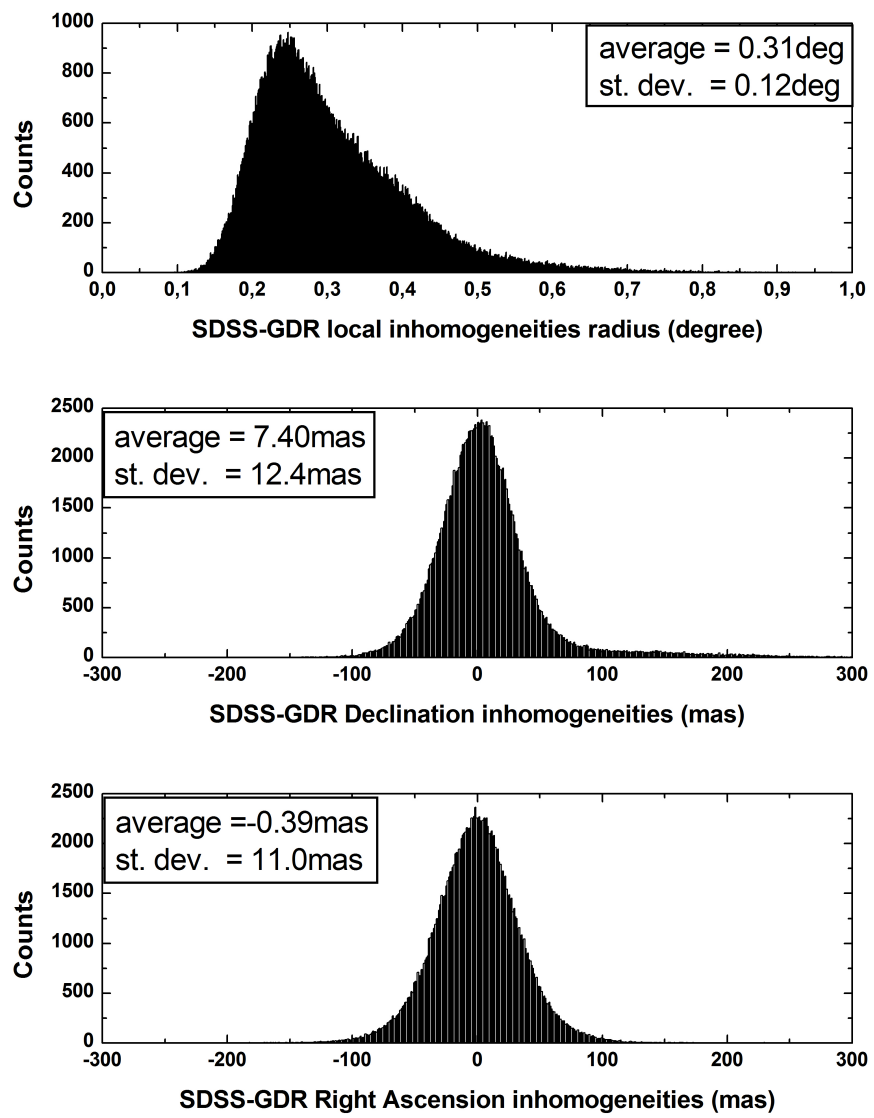

Fig. 10. Histograms for the local inhomogeneities corrections of the equatorial coordinates (already Euclidean rotations oriented) of the SDSS quasars relatively to the GDR2 positions. From bottom to top: corrections in right ascension, declination, and position.

corrections are indeed coherent, testifying the need for their removal. The two plots show how the standard deviation increases up to stabilise over about five degrees of distance. They are obtained by taking each quasar in turn as a pole source, and plotting the standard deviation in the inhomogeneity correction within growing distance rings. For nearby sources, that is, very small rings, the inhomogeneities are much the same, and the standard deviation is accordingly small. We note that already at the distance of five degrees, the standard deviation reaches the ceiling of scatter.

In the LQAC-5 there are 58881 sources that do not belong to the SDSS. Of these, 21903 do not belong either to the DR1. Therefore for 36978 QSOs, it is useful to determine a set of equatorial coordinates placed onto the (provisional) Gaia Celestial Reference Frame. As described above, that same goal was attained for the 172296 sources that belong to the SDSS but not to the GDR1.

For the non-SDSS sources, which are collected from many different catalogues, with as many different systematic astrometric biases, this is attained using the former LQRF equatorial coordinates referred to the ICRF2 as a common rigid initial reference frame. There are 35671 sources fulfilling the conditions above. For these sources, the steps towards the calculation of their DR1 referred LQRF coordinates are made using the 11770 sources that concurrently belong to the same original catalogues, as well as to the DR1 and to the former version ICRF2-referred LQRF.

The average difference to the DR1 position is 24.6 mas, that is 13.1 mas larger than the average position difference from the
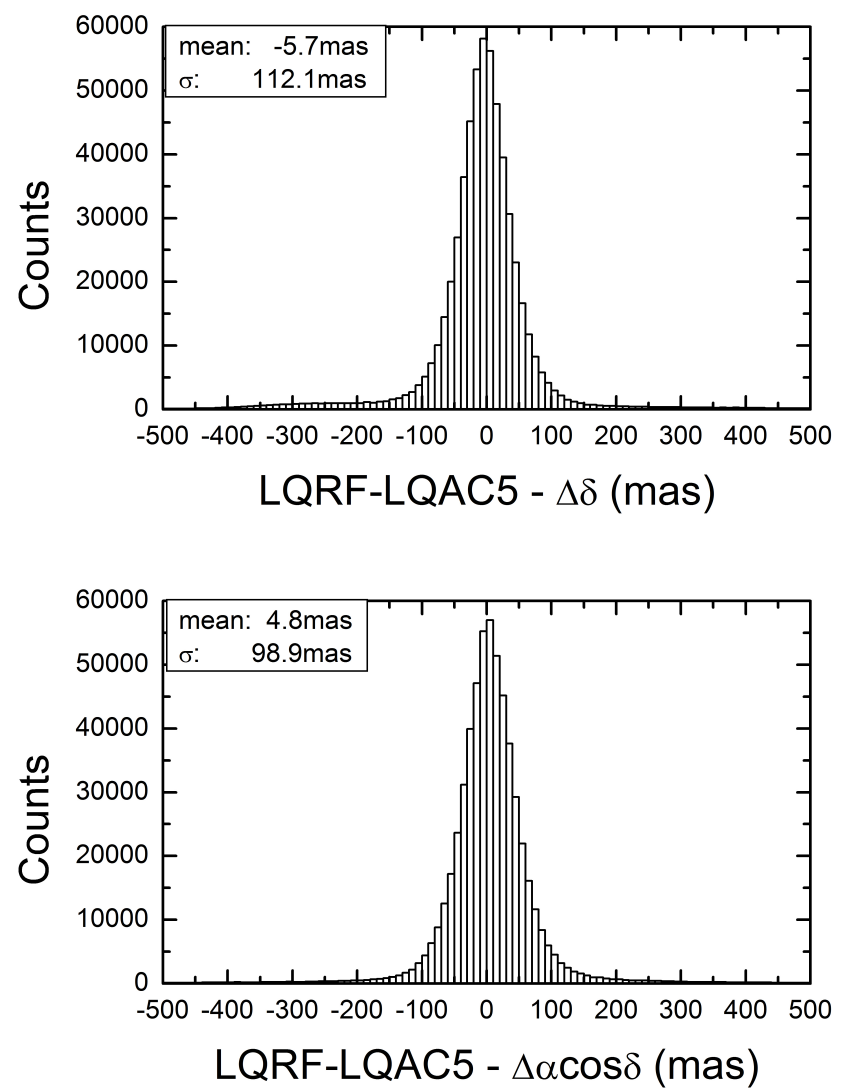

Fig. 11. Histograms comparing the LQRF coordinates referred to the Gaia data releases and the LQAC coordinates extracted from several catalogues.

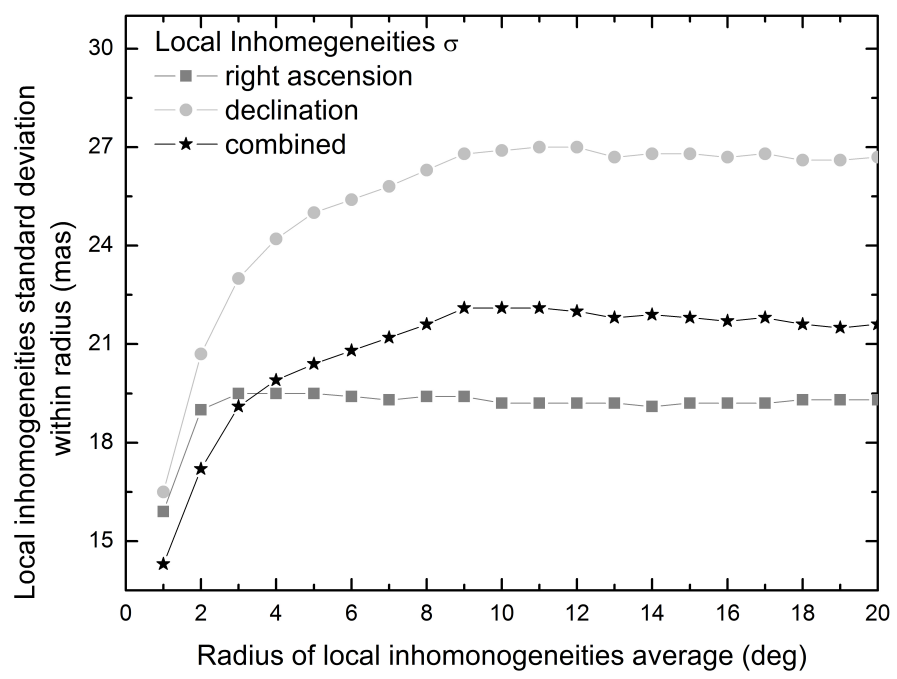

Fig. 12. Local inhomogeneities coherency over distance. The estimator is the average for all SDSS quasars of the standard deviation of the corrections as calculated in circles of increasing radii centred on each quasar.

SDSS to the DR1. The three Euclidean rotations and the equatorial offset are significant, though they are at a level similar to those found for the SDSS sources. This happens because they are distributed all over the sky, except across the direction of the Galaxy disc, while the SDSS concentrates on a quarter of the sky. As before, adopting the solution that acknowledges the more equations, the equinox bias is $-11.7 \pm 1.0$ mas and the equatorial bias is $+20.6 \pm 0.6$ mas. The rotation around the 
equinox oriented $X$-axis is $+23.9 \pm 0.6$ mas, and finally the rotation around the remaining triedal $Y$-axis is $+10.8 \pm 0.7$ mas. The overall standard deviation of the solution amounts to 104.9 mas.

Finally, the determination of the local inhomogeneities follow the same method as before. However, the number of sources is smaller and their distances larger. Notwithstanding the fact in only $1.3 \%$ of the cases the sources of comparison have to be found beyond ten degrees. For the right ascensions the average is +0.86 mas with a standard deviation of 10.48 mas, and extreme values of -66.0 mas and +89.6 mas. For the declination the average is -1.89 mas with a standard deviation of 11.24 mas, and extreme values of -74.7 mas and +78.8 mas.

As a result, the so formed LQRF for the non-SDSS sources diverges from the DR1 frame on average by $-7.25 \pm 12.16$ mas in right ascension and by $+12.86 \pm 20.55$ mas. In other words, the initial divergence is more than halved.

\section{Determination of absolute magnitudes}

As in the previous releases of the LQAC series, we give the absolute magnitudes of the quasars in the five SDSS bands $(u, g, r, i$, and $z$ ), when it is possible. The absolute magnitude quantifies the intrinsic luminosity of the quasars with respect to a given wavelength band. Consequently, absolute magnitudes are supposed to be unaffected by the cosmological redshift due to the expansion of the Universe. So their calculation requires a set of corrections to account for the different issues that affect light between the emitter and the observer.

For this purpose we applied the same method of computation used in the LQAC-4 (Coelho et al. 2019). With that method, absolute magnitudes are calculated with the following equation:

$M=m+5-5 \log \left(D_{\mathrm{L}}\right)-A-A_{\mathrm{IG}}-A_{\text {host }}-K$

where $m$ refers to the apparent magnitude of the quasar corresponding to the given bandwidth and $D_{\mathrm{L}}$ is the luminosity distance determined according to Hogg (1999). The four components $A, A_{\mathrm{IG}}, A_{\mathrm{host}}$, and $K$ account for corrections which characterise effects that can significantly affect the result if they are not accounted for in the calculation.

The first component, $A$, corrects the Galactic extinction due to dust of the Milky Way present on the quasar line of sight. Apparent SDSS magnitudes were therefore corrected from that effect using the classical maps of Schlegel et al. (1998). In future LQAC updates, more recent values could also be extracted from Schlafly \& Finkbeiner (2011). The second component, $A_{\mathrm{IG}}$, corrects the intergalactic (IG) light absorption associated with the Lyman $\alpha(\operatorname{Ly} \alpha)$ forest. More precisely, it consists essentially of Ly $\alpha(1215.67 \AA)$ resonance absorption lines in different redshifts (Lynds 1971), produced when the QSO's light passes through intergalactic clouds of neutral hydrogen (see Rauch 1998, and references therein).

The corresponding corrections were extracted from the Type $\mathrm{I}^{2}$ quasi-stellar object (QSO) curves of Meiksin (2006). The third correction, $A_{\text {host }}$, is relative to the quasar reddening caused by the dust in its host galaxy. The Small Magellanic Cloud "bar" (SMC "bar") extinction law has been validated as the best way to describe the reddening of large quasar populations (Hopkins et al. 2004; Krawczyk et al. 2015; Coelho et al. 2019). Thus, for the computation of this correction we followed

\footnotetext{
2 Type I QSO refers to active galactic nuclei that exhibit broad and narrow emission lines and are interpreted as being seen under an angle quite large with respect to the torus plane.
}

Richards et al. (2003) and Hopkins et al. (2004) approach considering the SMC bar extinction law from Gordon et al. (2003).

Finally, the absolute magnitude was obtained by taking into account a last correction, the so-called K-correction, which represents the redshift effect on the radiation observed in each bandpass, due to the expansion of the Universe. The main difference between our approach to obtain the absolute magnitudes and the preceding ones resides in the use of the Gaia library of synthetic spectra (Claeskens et al. 2006) to model the quasar spectral energy distribution (SED). This library allows us to use models with different intensities for the emission lines, and different values of spectral indices for the continuum. This method presents a more complete approach than the previous ones used in the literature. It gives significantly brighter magnitudes for high redshifts, in comparison with the simpler approach of Souchay et al. (2015) for the LQAC-3, due to the inclusion of the extra extinction corrections and the different SED model as demonstrated by Coelho et al. (2019). We note that with our available photometric data, ranging from the near-UV to the mid-IR, SED fitting starting from the spectroscopic redhift could be considered. It may also give accurate absolute magnitudes, and also give some information about quasar absorption, type, and BAL.

\section{Morphological indices}

Most of the quasar luminous output comes from the accretion disc, an inner region ranging the milli-arcsecond or smaller. Through physical processes and heating a small amount of light can also come from outer regions as optical jets shocks and blobs travelling along them, or from broad or narrow line emitting regions. Light from the host galaxy can be perceived for the nearby quasars. Because of the large discrepancy of the output and of the cosmological distances, a detailed map of the light distribution by direct observation is rare. Instead, here, the pointspread function of the quasars' image is used to establish a morphological index.

The concept is similar to the one used by SDSS (Gunn et al. 2006) and Milliquas (Flesch 2015), but our method employs IRAF photometry functions and compares the target's PSF against that of nearby stars. For each object, we extract fields of $5 \times 5 \mathrm{~s} \delta$ arc-minutes from the DSS (Digitized Sky Survey) plates B, R, and IR. The choice for the DSS continues what was done in previous versions of the LQAC, since it covers the entire sky and the plate emulsion sensitivity matches the results obtained in SDSS comparison fields (Andrei et al. 2012). The elements of comparison are the parameters SHARP, SROUND, and GROUND, determined by IRAF's DAOFIND on the Johnston $B, R$, and $I$ digitised plates of the DSS. The morphological indexes of each quasar are given by Andrei et al. (2008):

$M_{\mathrm{PC}}=\left|P_{\mathrm{Q}}-\overline{P_{\mathrm{s}}}\right| / \sigma_{\mathrm{s}}$

where $M_{\mathrm{PC}}$ is the morphological index of quasar $Q$ for the PSF parameter $P$ in the colour $C$, normalised by the standard deviation on parameter $P_{\mathrm{s}}$ given in comparison to the mean value from the stars $s$.

There are 149084 new quasars in the LQAC-5, which were retrieved in 119352 B plates, 148615 R plates, and 145536 IR plates. The missing fields correspond to faulty sky patches in the DSS. On top of this, quasars are missing by being too faint (most of cases) or in a very few cases by lack of suitable comparison stars. The comparison stars must conform to be at least ten pixels off the boundaries of the field, as well as isolated by five pixels. Under these provisions, three kinds of comparisons were made: 


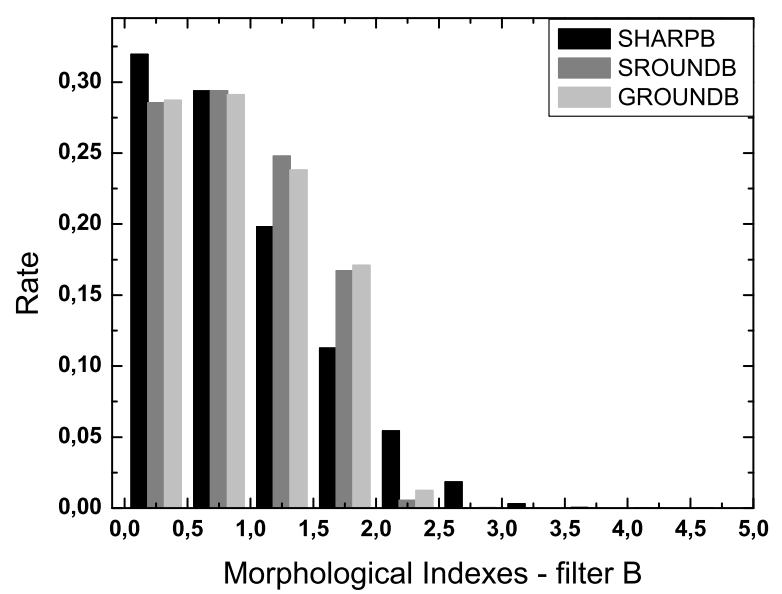

Fig. 13. Histogram of the morphological indices from images from the DSS filter B, for the new quasars of the LQAC-5 for which all the three morphological indices were determined. This corresponds to 48280 quasars. The mnemonic before the filter letter refers to the IRAF's DAOFIND SHARP, SROUND, and GROUND.

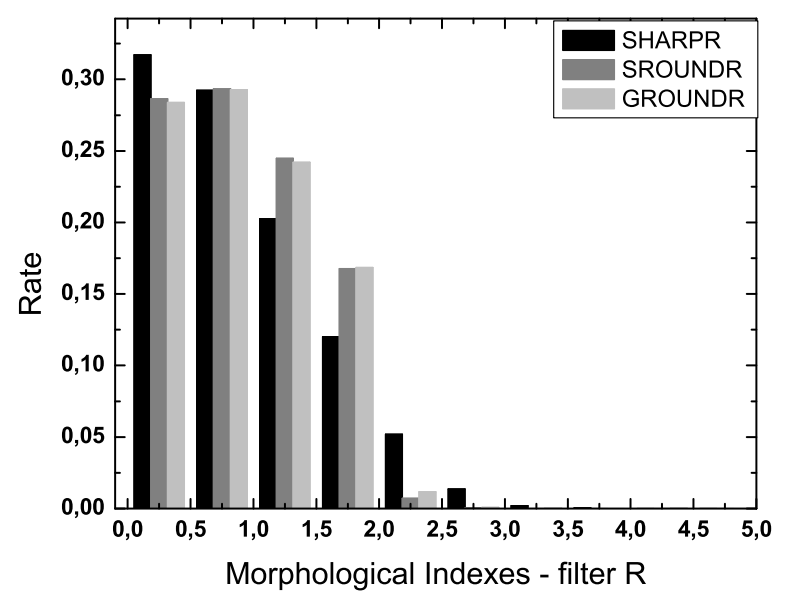

Fig. 14. Histogram of the morphological indexes from images from the DSS filter R for the new quasars of the LQAC-5 for which all the three morphological indices were determined. This corresponds to 88271 quasars. The mnemonic before the filter letter refers to the IRAF's DAOFIND SHARP, SROUND, and GROUND.

a first one against all the stars in the field, a second one against the ten stars closer to the target, and a third one against the ten stars closer and of comparable magnitude to the target. We found that the results in all three cases are fully equivalent. Thus the comparison with more stars was finally adopted.

For the new sources in the LQAC-5, all the morphological indexes were obtained for 24604 quasars. For 136420 quasars at least one morphological index was obtained. Likewise, in the B filter all morphological indices were obtained for 48280 quasars, and at least one morphological index for 51349 quasars; in the $\mathrm{R}$ filter all morphological indices were obtained for 88271 quasars, and at least one morphological index for 94342 quasars. In the IR filter all morphological indexes were obtained for 106971 quasars, and at least one morphological index for 113246 quasars. These numbers reflect that the majority of the new LQAC-5 objects originate from the Baryon Oscillation Spectroscopic Survey (BOSS, Dawson et al. 2013). This survey was designed to target faint quasars in the redshift range $z=2.2-3.5$ (Ross et al. 2012), and they are mostly unobscured objects.

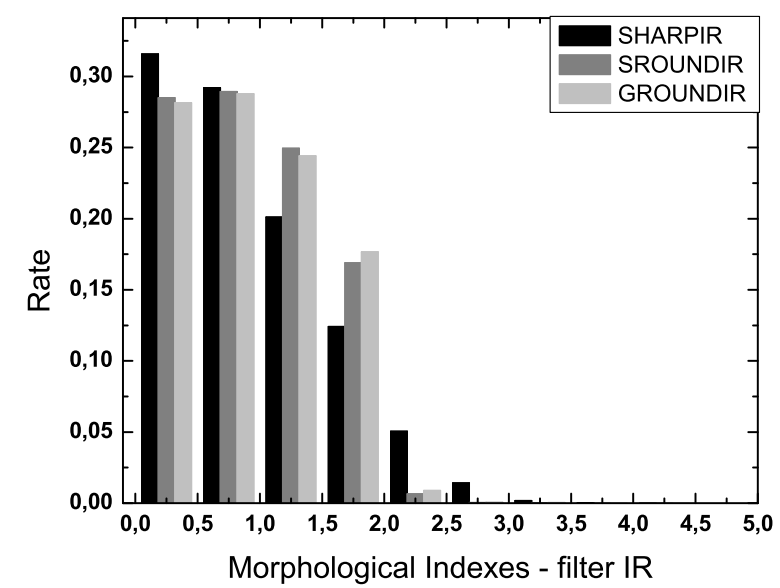

Fig. 15. Histogram of the morphological indexes from images from the DSS filter IR, for the new quasars of the LQAC-5 for which all the three morphological indices were determined. This corresponds to 106971 quasars. The mnemonic before the filter letter refers to the IRAF's DAOFIND SHARP,SROUND, and GROUND.

There are no magnitude or redshift dependences on any of the morphological indices. Figures $13-15$ present the percentage histograms of the SHARP, SROUND, and GROUND morphological indices, in the B, R, and IR filters. They resemble the analogous plots in the LQAC-4 (Gattano et al. 2018), which also contained a large fraction of BOSS-SDSS quasars. Now, however, the histograms for the three indexes are characteristically flat. There is a slight peculiarity of the SHARP index, which is in line with unobscured, bright centre quasars. Also the modal values for the indices is around 0.8 , and the percentage of indices larger than 2 is at $8 \%$, that is the new LQAC5 quasars are slightly more stellar-like than in the previous LQAC issues.

\section{LQAC-5 catalogue}

The LQAC-5 contains 592809 quasars which represents an increase of $33.59 \%$ with respect to the 443725 objects compiled in the LQAC-4. All the new objects are coming from the SDSS DR14Q release (Pâris et al. 2018). The individual catalogues providing source entries in the compilation are the same as in the LQAC-4. Moreover each one of them is represented by a flag (A to $\mathrm{M}$ ). The basic descriptions and specificities of each individual catalogue can be found in the previous papers related to the LQAC-2 (Souchay et al. 2012) and LQAC-3 (Souchay et al. 2015).

In Table 2, we show for each individual catalogue the number of cross-identifications of the objects with both the Gaia DR1 and the Gaia DR2 catalogues. The percentage of recognition with the DR2 range between $67 \%$ and $77 \%$ for the objects belonging to the radio VLBI catalogues (Flags " $A$ " to "D"), characterised by very accurate determinations of the equatorial coordinates. In particular for the ICRF2, the number of cross-identifications is increased by 310 objects from the DR1 to the DR2, which corresponds to a $9.08 \%$ increase. Otherwise, the most significant improvements of the number of detections between the two Gaia releases concern the LRG $(+21.92 \%)$ and the $2 \mathrm{QZ}(+17.37 \%)$. The FIRST catalogue presents a leading percentage of recognition with the DR2 (97.94\%) with only 20 lacking objects to be compared with a total of 969 units, whereas the lower percentage concerns the LRG with a rate smaller than $50 \%$.

In the following, with the help of Table 3, we present some new or improved characteristics of the LQAC-5 catalogue with respect to the LQAC-4 (Gattano et al. 2018). 
Table 2. Number of quasars present in each individual catalogue of the LQAC-5 compilation with the number of quasars in common with the Gaia DR1 and DR2 catalogues, with the corresponding percentage.

\begin{tabular}{|c|c|c|c|c|c|c|c|}
\hline Name & Ref. & Flag & $\mathrm{Nb}$. & $\mathrm{Nb}$. in DR1 & $\%$ in DR 1 & $\mathrm{Nb}$. in DR2 & $\%$ in DR2 \\
\hline ICRF2 & Fey et al. (2015) & A & 3414 & 2314 & 67.78 & 2624 & 76.86 \\
\hline VLBA & Found in RFC $2015 \mathrm{~d}^{(1)}$ & B & 7212 & 4287 & 59.44 & 4873 & 67.57 \\
\hline VLA & Found in VLA calibrator manual ${ }^{(2)}$ & $\mathrm{C}$ & 1858 & 1228 & 66.09 & 1412 & 75.99 \\
\hline JVAS & $\begin{array}{l}\text { Patnaik et al. (1992) } \\
+ \text { Browne et al. (1998) }\end{array}$ & $\mathrm{D}$ & $\begin{array}{r}2118(800) \\
(781)\end{array}$ & 1373 & 64.83 & 1572 & 74.22 \\
\hline SDSS & Pâris et al. (2018) & $\mathrm{E}$ & 548206 & (3) 218698 & (3) 56.82 & 368149 & 67.15 \\
\hline $2 \mathrm{QZ}$ & Croom et al. (2004) & $\mathrm{F}$ & 23654 & 18072 & 76.40 & 22180 & 93.77 \\
\hline FIRST & Becker et al. (1995) & $\mathrm{H}$ & 969 & 909 & 93.81 & 949 & 97.94 \\
\hline $\mathrm{HB}$ & Hewitt \& Burbidge (1993) & I & 6720 & 5991 & 89.15 & 6385 & 95.01 \\
\hline 2MASS & Cutri et al. (2003) & J & 21882 & 20338 & 92.94 & 21391 & 97.76 \\
\hline $\mathrm{GSC} 2.3$ & Lasker et al. (2008) & $\mathrm{K}$ & 144496 & 123012 & 85.13 & 137967 & 95.48 \\
\hline B1.0 & Monet et al. (2003) & $\mathrm{L}$ & 139177 & 121446 & 87.26 & 135101 & 97.07 \\
\hline VV & Véron-Cetty \& Véron (2010) & $\mathrm{M}$ & $14506^{(4)}$ & 4180 & 28.82 & 4696 & 32.38 \\
\hline
\end{tabular}

Notes. ${ }^{(1)}$ Publicly available at http://alt.astrogeo.org/rfc/. ${ }^{(2)}$ Publicly available at http://www.vla.nrao.edu/astro/calib/ manual/index.shtml. ${ }^{(3)}$ The 218698 objects and the corresponding percentage are the results of the crossmatches between the 384834 SDSS DR12Q objects of the LQAC-4 with the DR1. ${ }^{(4)}$ These 14506 objects are those from Véron-Cetty \& Véron (2010) which do not belong to any of the catalogues with flags "A" to "I".

Table 3. Number of entries per item in the LQAC-5, with the corresponding percentage.

\begin{tabular}{lrr}
\hline \hline Item & Number of objects & Percentage \\
\hline Redshift $z$ & 589364 & 99.42 \\
DR2 detection only & 149935 & 25.29 \\
DR1 and DR2 detections & 248365 & 41.90 \\
DR1 detection only $(\alpha, \delta)$ & 397 & 0.07 \\
parallax in DR2 & 319905 & 53.96 \\
proper motion $\left(\mu_{\alpha}, \mu_{\delta}\right)$ in DR2 & 319905 & 53.96 \\
$G$ (DR2) & 398300 & 67.19 \\
$G$ (DR1) & 248762 & 41.96 \\
BP & 370702 & 62.53 \\
RP & 370738 & 62.54 \\
$u$ (SDSS and others) & 572299 & 96.54 \\
$g$ (SDSS only) & 540617 & 91.20 \\
$r$ (SDSS and others) & 570303 & 96.20 \\
$i$ (SDSS and others) & 555460 & 93.69 \\
$z$ (SDSS only) & 540620 & 91.20 \\
$J$ (2MASS) & 21882 & 3.69 \\
$K$ (2MASS) & 21882 & 3.69 \\
$W_{1}$ (AllWISE) & 488736 & 82.44 \\
$W_{2}$ (AllWISE) & 488736 & 82.44 \\
$W_{3}$ (AllWISE) & 488599 & 82.42 \\
$W_{4}$ (AllWISE) & 488597 & 82.42 \\
Radio Flux at $1.4 \mathrm{Ghz}$ & 11594 & 1.96 \\
Radio Flux at $2.3 \mathrm{Ghz}$ & 4268 & 0.72 \\
Radio Flux at $5.0 \mathrm{Ghz}$ & 5340 & 0.90 \\
Radio Flux at $8.3 \mathrm{Ghz}$ & 6465 & 1.09 \\
Radio Flux at $24.0 \mathrm{Ghz}$ & 61 & 0.01 \\
Absolute magnitude $M_{B}$ & 152864 & 25.79 \\
Absolute magnitude $M_{I}$ & 283682 & 47.85 \\
Absolute magnitude $M_{u}$ & 525523 & 88.65 \\
Absolute magnitude $M_{g}$ & 525523 & 88.65 \\
Absolute magnitude $M_{r}$ & 525523 & 88.65 \\
Absolute magnitude $M_{i}$ & 525523 & 88.65 \\
Absolute magnitude $M_{z}$ & 525523 & 88.65 \\
\hline & & \\
& & \\
& &
\end{tabular}

Table 4. Origin of the coordinates chosen for the LQAC-5 with respect to those chosen for the LQAC-4

\begin{tabular}{lrr}
\hline \hline LQAC coord. origin & LQAC-4 & LQAC-5 \\
\hline Gaia DR2 & 0 & 398300 \\
Gaia DR1 & 246474 & 394 \\
ICRF & 3414 & 793 \\
LQRF & 119374 & 183696 \\
ORIG & 74463 & 9626 \\
\hline Total & 443725 & 592809 \\
\hline
\end{tabular}

Improvement of determination of the equatorial coordinates. LQAC-5 gives the equatorial coordinates in the original catalogue given by the first positive flag (A to $\mathrm{M}$ ) in the alphabet order, which corresponds to an a priori decreasing order of accuracy, as in the previous versions of the LQAC. Moreover, the LQAC-5 is an astrometric catalogue which gives separately the a priori best determination of equatorial coordinates following the decreasing hierarchical order of accuracy: Gaia DR2 $>$ ICRF2 $>$ LQRF $>$ ORIGINAL. This means that when a quasar belongs to the Gaia DR2 release, its coordinates are chosen in priority, even if it belongs to the ICRF2. If the quasar belongs to the ICRF2 but is not present in the DR2, the ICRF2 coordinates are chosen. When a quasar is not identified either in the DR2 or as an ICRF component, there are two remaining possibilities: if it has LQRF coordinates, they are chosen in priority. In the negative case, there is no other choice than keeping the original coordinates. The quantitative results concerning these choices are shown in Table 4. A significant improvement lies in the important decrease of original coordinates (flagged as "ORIGIN") in favour of LQRF coordinates, which means an a priori better estimation of these coordinates, as detailed in Sect. 5. Thus the number of original coordinates dropped drastically from 74463 to 9626 .

The LQAC membership flag. In the LQAC-5 catalogue, a flag ("3", "4", or "5"), is indicated for each entry to specify the first appearance of the quasar to the successive up-dates of the 
LQAC, that is, respectively the LQAC-3 (Souchay et al. 2015), the LQAC-4 (Gattano et al. 2018) and the LQAC-5 (this paper).

Parallaxes and proper motions from Gaia DR2. These two items constitute a very important additional piece of information in the LQAC-5 up-date with respect to the LQAC-4 one. The reason is that this fundamental data was not present in the DR1 whereas it appeared for the first time in the DR2 release. We note that these two parameters are completely dependent, in other words, there is no case for which the parallax is given without proper motion and vice versa. Moreover, we can note that this data concerns 319905 objects. That corresponds to $80.25 \%$ of the total number of quasars present either in the DR1 or in the DR2, which amounts to 398697 units. This corresponds to $53.96 \%$ of the whole LQAC-5 sample.

Mid-infrared data. For the first time in a LQAC update, we have carried out the cross-identification with the AllWISE survey (Wright et al. 2010), which brings mid-infrared magnitudes $W_{1}$ and $W_{2}$ for 488736 objects, $W_{3}$ for 488599 ones $W_{4}$ for 488507 ones. This corresponds respectively to $82.44 \%, 82.42 \%$ and $82.40 \%$ of the overall LQAC-5 population. This result is consistent with the completeness estimate made by Secrest et al. (2015, see Sect. 4.4 therein).

\section{Conclusion}

In this paper, we have elaborated an updated version of the Large Quasar Astrometric Catalogue, namely the LQAC-5, by adding exclusively new quasars coming from the DR14Q release of the SDSS (Pâris et al. 2018). Thus, the total number of recorded quasars in our compilation reaches 592809 objects. This constitutes a $33.59 \%$ increase with respect to the previous LQAC-4 version (Gattano et al. 2018), which, with its 443725 objects, presented a $37.82 \%$ increase with respect to the LQAC-3 (Souchay et al. 2015).

In addition to the 149084 new entries, an important advantage of the LQAC-5 with respect to the LQAC-4 is the crossidentification with the Gaia DR2 catalogue, which enables us in particular to reckon undoubtedly 398300 Gaia objects as known quasars. This figure represents $67.18 \%$ of the whole population in LQAC-5. This can be compared with the 249071 Gaia DR1 objects recognised in the LQAC-4 catalogue, which represents a $59.91 \%$ increase. This quantitative improvement is accompanied by a remarkable refinement of the source position quality, in other words, the improvement of the equatorial position uncertainties for quasars observed by Gaia. In a very large majority, the Gaia DR2 data are more accurate than the Gaia DR1 data which have served for the LQAC-4. In an important proportion of cases, this is characterised by an improvement of a factor of ten.

Another important benefit of the cross-identification with Gaia DR2 lies in two fundamental new sets of parameters available for common quasars. These are the parallaxes and the proper motions in both equatorial coordinates. In general, given their distances, quasars present undetectable parallaxes and proper motions. That point constitutes a postulate, checked in VLBI, for instance when we refer to the ICRF2 (Fey et al. 2015). Therefore, the nominal values of the parallaxes and twodimensional proper motions values as given by Gaia DR2 should be coherent with a zero value when taking into account the value of the uncertainty.

As mentioned in the introduction, the LQAC-5 compilation induces naturally some comparisons with the very recent catalogues of AGN's elaborated both by Paine et al. (2018) and Assef et al. (2018). The first paper presents an all-sky sample of 567721 AGNs in Gaia Data Release 1, selected using WISE two-colour criteria. The catalogue has the advantage to propose a fairly uniform sky coverage beyond the Galactic plane, with a mean density of 12.8 AGNs per square degree and a magnitude limit at $G=20.7$. The AGN were obtained by cross-matching the AllWISE (Secrest et al. 2015) catalogue of MIR (mid infra-red) AGNs with Gaia Data Release 1 using the pre-computed WISE cross-match table provided in the Gaia archive (Marrese et al. 2017). The second paper presents two large catalogues of AGN candidates (R90 and C75) identified across $30093 \mathrm{deg}^{2}$ of the extragalactic sky again from AllWISE Data Release. Both catalogues are selected purely using the WISE W1 and W2 bands. The R90 catalogue consists of 4543530 AGN candidates with 90\% reliability, while the C75 catalogue consists of 20907127 AGN candidates with $75 \%$ completeness. From these specificities, we observe that the number of quasars in the first study is roughly equivalent to the number of objects in the LQAC-5 (592 809) whereas it corresponds to a ratio 7.6 to 1 and 35.3 to 1 respectively for the R90 and C75 surveys.

Nevertheless, the advantage of the LQAC-5 lies in two fundamental aspects: first, it gives a sample of objects duly recognised spectroscopically as quasars, excepted a very small population consisting of VLBI sources (among which ICRF2 ones) kept for their obvious astrometric quality. Second, as is illustrated by the acronym LQAC, it gives the best a priori determination of the celestial coordinates of each object, which is, for a large majority of objects, considerably more accurate than the determination of coordinates coming from the original catalogues. The LQAC is continuously improved and updated at the fast rhythm of large survey observations, for example, the next SDSS public release containing new eBOSS data is scheduled for the summer of 2019 and will contain spectroscopic data after four years of observations, which should represent more than 700000 quasars.

Acknowledgements. This work has been supported by a CNES post-doctoral grant. BC acknowledges support from the Advanced EU Network of Einfrastructures for Astronomy with SKA (AENEAS), funded by the European Commission Framework Programme Horizon 2020 RIA under grant agreement n. 731016 and from the ENGAGE SKA RI, grant POCI-01-0145-FEDER022217, funded by COMPETE 2020 and FCT, Portugal.

\section{References}

Andrei, A. H., Filho, V. A., Martins, R. V., et al. 2008, in A Giant Step: from Milli- to Micro-arcsecond Astrometry, eds. W. J. Jin, I. Platais, \& M. A. C. Perryman, IAU Symp., 248, 104

Andrei, A. H., Souchay, J., Zacharias, N., et al. 2009, A\&A, 505, 385

Andrei, A. H., Anton, S., Barache, C., et al. 2012, in SF2A-2012: Proceedings of the Annual meeting of the French Society of Astronomy and Astrophysics, eds. S. Boissier, P. de Laverny, N. Nardetto, et al., 61

Arenou, F., Luri, X., Babusiaux, C., et al. 2017, A\&A, 599, A50

Arias, E. F., Feissel, M., \& Lestrade, J. F. 1988, A\&A, 199, 357

Arias, E. F., Charlot, P., Feissel, M., \& Lestrade, J.-F. 1995, A\&A, 303, 604

Assef, R. J., Stern, D., Noirot, G., et al. 2018, ApJS, 234, 23

Becker, R. H., White, R. L., \& Helfand, D. J. 1995, ApJ, 450, 559

Browne, I. W. A., Wilkinson, P. N., Patnaik, A. R., \& Wrobel, J. M. 1998, MNRAS, 293, 257

Claeskens, J.-F., Smette, A., Vandenbulcke, L., \& Surdej, J. 2006, MNRAS, 367, 879

Coelho, B., Andrei, A., \& Anton, S. 2019, MNRAS, submitted

Croom, S. M., Smith, R. J., Boyle, B. J., et al. 2004, MNRAS, 349, 1397

Cutri, R. M., Skrutskie, M. F., van Dyk, S., et al. 2003, 2MASS All Sky Catalog of Point Sources (NASA/IPAC Infrared Science Archive)

da Ângela, J., Shanks, T., Croom, S. M., et al. 2008, MNRAS, 383, 565

Dawson, K. S., Schlegel, D. J., Ahn, C. P., et al. 2013, AJ, 145, 10

Delchambre, L. 2018, MNRAS, 473, 1785

Eisenstein, D. J., Weinberg, D. H., Agol, E., et al. 2011, AJ, 142, 72

Evans, D. W., Riello, M., De Angeli, F., et al. 2017, A\&A, 600, A51 
Fey, A. L., Gordon, D., Jacobs, C. S., et al. 2015, AJ, 150, 58 Flesch, E. W. 2015, PASA, 32, e010

Gaia Collaboration (Prusti, T., et al.) 2016a, A\&A, 595, A1

Gaia Collaboration (Brown, A. G. A., et al.) 2016b, A\&A, 595, A2

Gaia Collaboration (Brown, A. G. A., et al.) 2018, A\&A, 616, A1

Gattano, C., Souchay, J., \& Barache, C. 2014, A\&A, 564, A117

Gattano, C., Lambert, S. B., \& Bizouard, C. 2017, J. Geodesy, 91, 849

Gattano, C., Andrei, A. H., Coelho, B., et al. 2018, A\&A, 614, A140

Gordon, K. D., Clayton, G. C., Misselt, K. A., Landolt, A. U., \& Wolff, M. J. 2003, ApJ, 594, 279

Gunn, J. E., Siegmund, W. A., Mannery, E. J., et al. 2006, AJ, 131, 2332

Hewitt, A., \& Burbidge, G. 1993, ApJS, 87, 451

Hogg, D. W. 1999, ArXiv e-prints [arXiv:astro-ph/9905116]

Hopkins, P. F., Strauss, M. A., Hall, P. B., et al. 2004, AJ, 128, 1112

Jordi, C., Gebran, M., Carrasco, J. M., et al. 2010, A\&A, 523, A48

Krawczyk, C. M., Richards, G. T., Gallagher, S. C., et al. 2015, AJ, 149, 203

Lasker, B. M., Lattanzi, M. G., McLean, B. J., et al. 2008, AJ, 136, 735

Le Poncin-Lafitte, C., Hees, A., \& Lambert, S. 2016a, Phys. Rev. D, 94, 125030

Le Poncin-Lafitte, C., Bourgoin, A., Hees, A., et al. 2016b, ArXiv e-prints [arXiv:1607.07394]

Lindegren, L., Lammers, U., Bastian, U., et al. 2016, A\&A, 595, A4

Lindegren, L., Hernández, J., Bombrun, A., et al. 2018, A\&A, 616, A2

Lynds, R. 1971, ApJ, 164, L73

Marrese, P. M., Marinoni, S., Fabrizio, M., \& Giuffrida, G. 2017, A\&A, 607, A105

Meiksin, A. 2006, MNRAS, 365, 807

Mignard, F., Klioner, S. A., Lindegren, L., et al. 2018, A\&A, 616, A14
Monet, D. G., Levine, S. E., Canzian, B., et al. 2003, AJ, 125, 984 Padovani, P., Alexander, D. M., Assef, R. J., et al. 2017, A\&ARv, 25, 2 Paine, J., Darling, J., \& Truebenbach, A. 2018, ApJS, 236, 37 Pâris, I., Petitjean, P., Aubourg, É., et al. 2014, A\&A, 563, A54 Pâris, I., Petitjean, P., Ross, N. P., et al. 2017, A\&A, 597, A79 Pâris, I., Petitjean, P., Aubourg, É., et al. 2018, A\&A, 613, A51 Patnaik, A. R., Browne, I. W. A., Wilkinson, P. N., \& Wrobel, J. M. 1992, MNRAS, 254, 655

Proft, S., \& Wambsganss, J. 2015, A\&A, 574, A46

Rauch, M. 1998, ARA\&A, 36, 267

Richards, G. T., Hall, P. B., Vanden Berk, D. E., et al. 2003, AJ, 126, 1131

Rosat, S., Lambert, S. B., Gattano, C., \& Calvo, M. 2017, Geophys. J. Int., 208, 211

Ross, N. P., Myers, A. D., Sheldon, E. S., et al. 2012, ApJS, 199, 3

Schlafly, E. F., \& Finkbeiner, D. P. 2011, ApJ, 737, 103

Schlegel, D. J., Finkbeiner, D. P., \& Davis, M. 1998, ApJ, 500, 525

Secrest, N. J., Dudik, R. P., Dorland, B. N., et al. 2015, ApJS, 221, 12

Souchay, J., Andrei, A. H., Barache, C., et al. 2009, A\&A, 494, 799

Souchay, J., Andrei, A. H., Barache, C., et al. 2012, A\&A, 537, A99

Souchay, J., Andrei, A. H., Barache, C., et al. 2015, A\&A, 583, A75

Urry, C. M., \& Padovani, P. 1995, PASP, 107, 803

Véron-Cetty, M.-P., \& Véron, P. 2010, A\&A, 518, A10

Wilkinson, P. N., Browne, I. W. A., Patnaik, A. R., Wrobel, J. M., \& Sorathia, B. 1998, MNRAS, 300, 790

Wright, E. L., Eisenhardt, P. R. M., Mainzer, A. K., et al. 2010, AJ, 140, 1868

York, D. G., Adelman, J., Anderson, Jr., J. E., et al. 2000, AJ, 120, 1579

Zacharias, N., Finch, C. T., Girard, T. M., et al. 2013, AJ, 145, 44 